Session 3B: STARS

Opacity driving, levitation, opacity data 


\title{
The solar chemical composition
}

\author{
N. Grevesse ${ }^{1,2}$ \\ ${ }^{1}$ Centre Spatial de Liège, Université de Liège, Avenue Pré Aily, B-4031 Angleur-Liège, Belgium \\ 2 Institut d'Astrophysique et de Géophysique, Université de Liège, \\ Allée du 6 Août, 17, B-4000 Liège, Belgium
}

\begin{abstract}
We give a brief review on the solar abundances emphasizing "hot topics" like the abundances of oxygen and neon.

\section{Introduction}

With Martin Asplund (Max Planck Institute of Astrophysics, Garching) and Jacques Sauval (Observatoire Royal de Belgique, Brussels) we recently published detailed reviews on the solar chemical composition (Asplund et al. 2005, Grevesse et al. 2007). These new abundances result essentially from the use of a 3D hydrodynamic model of the solar atmosphere instead of the classical 1D hydrostatic models, accounting for departures from LTE when possible, and the use of improved atomic and molecular data for many different indicators of the abundances. These new abundances are significantly smaller than previously recommended values at least for the most abundant metals, resulting in a decrease of the solar metallicity. While resolving a number of longstanding problems, the new solar abundances are in contrast with the Standard Solar Model. Models computed with the new abundances disagree with the very precise measurements of the sound speed profile, the abundance of $\mathrm{He}$ in the convection zone and the depth of this convection zone as derived from helioseismology. The abundances of $\mathrm{O}, \mathrm{C}$ and Ne do play key roles as major contributors to the opacities in the layers below the convection zone. As new works have recently appeared on $\mathrm{O}$ and $\mathrm{Ne}$, we propose to discuss them in more details hereafter.
\end{abstract}

\section{The solar abundance of oxygen}

Thanks to the recipe mentioned above, Asplund et al. (2004), Scott et al. (2006) and Meléndez (2004) have reanalyzed the solar abundance of oxygen from all the indicators available, permitted and forbidden atomic lines as well as the $\mathrm{CO}$ vibration-rotation lines and the $\mathrm{OH}$ vibration-rotation and pure rotation lines in the infrared solar spectrum. The new solar abundance of $\mathrm{O}$ is $\mathrm{A}(\mathrm{O})=8.66$, in the usual astronomical scale. This value is much lower, by about 0.2 dex, than previously recommended values. A series of new papers on the solar abundance of $\mathrm{O}$ has been published recently. We comment on these papers hereafter.

Ayres et al. (2006) also analyzed the CO lines with quite a different result. Their analysis used various empirical 1D models and they found a high value of the abundance of $O$. This is quite normal because the $\mathrm{CO}$ lines are extremely sensitive to temperature. The 3D effects are very large on such lines as shown by Scott et al. (2006), decreasing the abundance found 
when using 1D models, by rather large amounts. The best 1D model, for example a mean 3D model, could never simulate the 3D effects which are nonlocal and nonlinear.

Socas-Navarro \& Norton (2007) confirmed the low abundance of O . They constructed an empirical 3D model from spatially resolved spectropolarimetric observations of spectral lines and applied it to the analysis of the infrared triplet of $\mathrm{O} I$ at $777 \mathrm{~nm}$. However Centeno \& Socas-Navarro (2008) analyzing in sunspots, the forbidden $O$ I line at $630 \mathrm{~nm}$, blended with a $\mathrm{Ni} I$ line, derived a much larger abundance of $\mathrm{O}, \mathrm{A}(\mathrm{O})=8.86$. If this result is updated for a better transition probability of the [O I] line $(-0.06$ dex $)$, for a better solar abundance of $\mathrm{Ni}(-0.06 \mathrm{dex})$ and for a more precise effect of the formation of $\mathrm{CO}$ in sunspots $(-0.08$ dex $)$, the resulting abundance of $O$ becomes $A(O)=8.66$, in agreement with our low value.

Very recently, Ayres (2008) carefully analyzed the same forbidden O I line, using profile fits with a $3 \mathrm{D}$ model atmosphere. He got a high abundance of $\mathrm{O}, \mathrm{A}(\mathrm{O})=8.81$. But the best fits of the profiles are obtained if the $\mathrm{Ni} I$ blend is treated as a free parameter. This results in a too small contribution of $\mathrm{Ni}$ to the blend. If this contribution is increased to its normal value, obtained with the new abundance of $\mathrm{Ni}$ we have derived from a new analysis (to be published soon) with a 3D model, the abundance of $O$ can decrease to $A(O)=8.74$ i.e. very near to our value (see hereafter). The agreement between observed and computed profiles however degrades if the $\mathrm{Ni}$ abundance increases.

The most important new analysis of the solar abundance of $O$ has been recently done by Caffau et al. (2008). These authors analyze very carefully the permitted and forbidden lines of $\mathrm{O} I$ on various solar atlases observed at the center of the solar disc and on the whole disc. They use different 1D models as well as their own 3D original model. They recommend a rather high abundance of $\mathrm{O}, \mathrm{A}(\mathrm{O})=8.79$. One of the main differences between this work and previous works is to be found in the equivalent widths of the lines. The data from Caffau et al. are always larger than any other measurement by other authors. We remeasured very carefully these equivalent widths and never succeeded to reach their values. For example, for the infrared triplet at $777 \mathrm{~nm}$, we systematically found equivalent widths smaller by about 4 percent. We therefore normalysed the Caffau et al. (2008) results as well as our own results of Asplund et al. (2004) to the new equivalent widths and found abundances of $O$ in pretty good agreement, $A(O)=8.71$ versus $A(O)=8.70$.

We shall therefore recommend $A(O)=8.70$ as the solar abundance of $O$. It is also this low value which is found by Meléndez \& Asplund (2008) from the analysis of the third forbidden line of $\mathrm{O} \mathrm{I}$ at $557.7 \mathrm{~nm}$. This line is however heavily perturbed by two lines of $\mathrm{C}_{2}$.

New works are going on, based on new 3D models. Results are to be expected quite soon.

\section{The solar abundance of neon}

As no spectral line of neon is present in the solar photospheric spectrum, the solar abundance of neon is generally derived from the ratio $\mathrm{Ne} / \mathrm{O}$ measured in coronal matter; see e.g. Grevesse et al. (2007) for a review. A ratio $\mathrm{Ne} / \mathrm{O}=0.15$ is usually adopted as representative of the coronal ratio. With the here above recommended abundance of $\mathrm{O}$, the solar abundance of $\mathrm{Ne}$ becomes $\mathrm{A}(\mathrm{Ne})=7.88$. Drake \& Testa (2005) and very recently Garcia Alvarez et al. (2008) suggested, from the analysis of mostly very active stars, that the solar $\mathrm{Ne} / \mathrm{O}$ should be much larger than 0.15 , with a value up to 0.41 , therefore increasing the solar $\mathrm{Ne}$ to $\mathrm{A}(\mathrm{Ne})=8.32$. We do believe that the stars considered in these analyses have nothing to do with stars of the solar type. The stars analyzed have activities that are 1 to 4 orders of magnitude larger than the sun. In such stars, as Güdel (2004) already suggested, the ratio $\mathrm{Ne} / \mathrm{O}$ can be enhanced naturally by a rather large factor over the solar ratio. This has also been recently confirmed by Robrade et al. (2008), who show that the ratio $\mathrm{Ne} / \mathrm{O}$ definitely increases when increasing the stellar activity from the solar activity level. 
Two recent papers also found higher solar Ne abundances. Landi et al. (2007) derived a high solar abundance of $\mathrm{Ne}, \mathrm{A}(\mathrm{O})=8.11$, from the analysis of the UV spectrum of a solar flare. One way to explain this high $\mathrm{Ne}$ could be related to the discussion we had here above: matter in an active medium like solar flare shows an increase in the $\mathrm{Ne} / \mathrm{O}$ ratio related to the difference in first ionization potentials between the two species (Ne: $21.6 \mathrm{eV}$ and O: $13.6 \mathrm{eV}$ ). Bochsler (2007) derived the $\mathrm{O}$ and $\mathrm{Ne}$ abundances from very variable solar wind data, by comparing the abundances of these species with $\mathrm{He}$. He also found high values for the abundances of both $\mathrm{O}$ (8.87) and $\mathrm{Ne}$ (7.96) with rather large uncertainties (0.12 dex). However his results depend crucially on the ratio $\mathrm{He} / \mathrm{H}$, very variable in the solar wind, and always much smaller than the solar convection zone value. Had he adopted a slightly smaller $\mathrm{He} / \mathrm{H}$ ratio, as reasonable as the one he adopted, the $\mathrm{O}$ and $\mathrm{Ne}$ abundances would have decreased down to the low values.

In the galactic medium around us, neon has been derived from B stars. These stars should have about the same $\mathrm{Ne}$ abundance as the sun because, in the galactic chemical evolution, $\mathrm{Ne}$ does not change much during the last few gigayears. Cunha et al. (2006) found a very large abundance of $\mathrm{Ne}$ in $\mathrm{B}$ stars in the Orion association, $\mathrm{A}(\mathrm{O})=8.11$. Recently however, Morel \& Butler (2008) derived a much lower abundance of $\mathrm{Ne}, \mathrm{A}(\mathrm{Ne})=7.97$, from a sample of nearby B-stars. The main uncertainties in these analyses come from the larger non-LTE effects and from the uncertainties in the effective temperatures. If we take these uncertainties into account, this last result is in good agreement with the value of $A(\mathrm{Ne})=7.88$ we would recommend for the solar abundance of neon.

\section{Conclusions}

The discussions here above show that the "low" solar abundances of oxygen and neon are robust. We however suggested that we should increase the values recommended in previous works by 10 percent i.e. $A(O)=8.70$ and $A(N e)=7.88$. With these abundances, the present solar metallicity, $Z$, has to be increased by 5 percent and becomes $Z=0.0128$. These "new" abundances of $\mathrm{O}$ and $\mathrm{Ne}$ are still much too low to allow solving the problem of the disagreement between the Standard Solar Models and helioseismology.

Acknowledgments. We thank Martin Asplund, Jacques Sauval, also Pat Scott and many others, too numerous to be cited, for this marvelous adventure in the revision of the solar chemical composition.

\section{References}

Asplund, M., Grevesse, N., \& Sauval, A. J. 2005, in Cosmic Abundances as Records of Stellar Evolution and Nucleosynthesis, ed. T.G. BarnesIII, F.N. Bash, ASP Conf. Ser. 336, 25

Asplund, M., Grevesse, N., Sauval, A. J., et al. 2004, A\&A, 417, 751

Ayres, T. R. 2008, ApJS, in press

Ayres, T. R., Plymate, C., \& Keller, C. U. 2006, ApJS, 165, 618

Bochsler, P. 2007, A\&A, 471, 315

Caffau, E., Ludwig, H.-G., Steffen, M., et al. 2008, A\&A, 488,1031

Centeno, R., \& Socas-Navarro, H. 2008, ApJ, 682, 61

Cunha, K. , Hubeny, I., \& Lanz, T. 2006, ApJ, 647, L143

Drake, J. J., \& Testa, P. 2005, Nature, 436, 525

Garcia Alvarez, D., Drake, J. J., \& Testa, P. 2008, ApJ, in press

Grevesse, N., Asplund, M., \& Sauval, A. J. 2007, SSRV., 130, 105

Güdel, M. 2004, A\&AR 12, 71 
Landi, E., Feldman, U., \& Doschek, G. A. 2007, ApJ, 659, 743

Meléndez, J. 2004, ApJ, 615, 1042

Meléndez, J., \& Asplund, M. 2008, A\&A, 490, 817

Morel, T., \& Butler, K. 2008, A\&A, 487, 307

Robrade, J., Schmitt, J. H. M. M., \& Favata, F. 2008, A\&A, 486, 995

Scott, P., Asplund, M., Grevesse, N., \& Sauval, A. J. 2006, A\&A, 456, 675

Socas-Navarro, H. \& Norton, A. A. 2007, ApJ, 660, L153

\section{DISCUSSION}

Aerts: So far, the pulsational line broadening in the sun has been ignored in fitting line profiles. While the mode amplitudes are low (of the order of $\mathrm{cm} / \mathrm{s}$ ) their collective effect might be of importance in deriving high-precision abundances. Has anyone investigated that effect?

Grevesse: I am not sure that this effect has ever been quantified However the velocities explained by convection are of the order of $1.5 \mathrm{~km} / \mathrm{s}$. So, I believe that the combined effect of the oscillations will be much smaller than this.

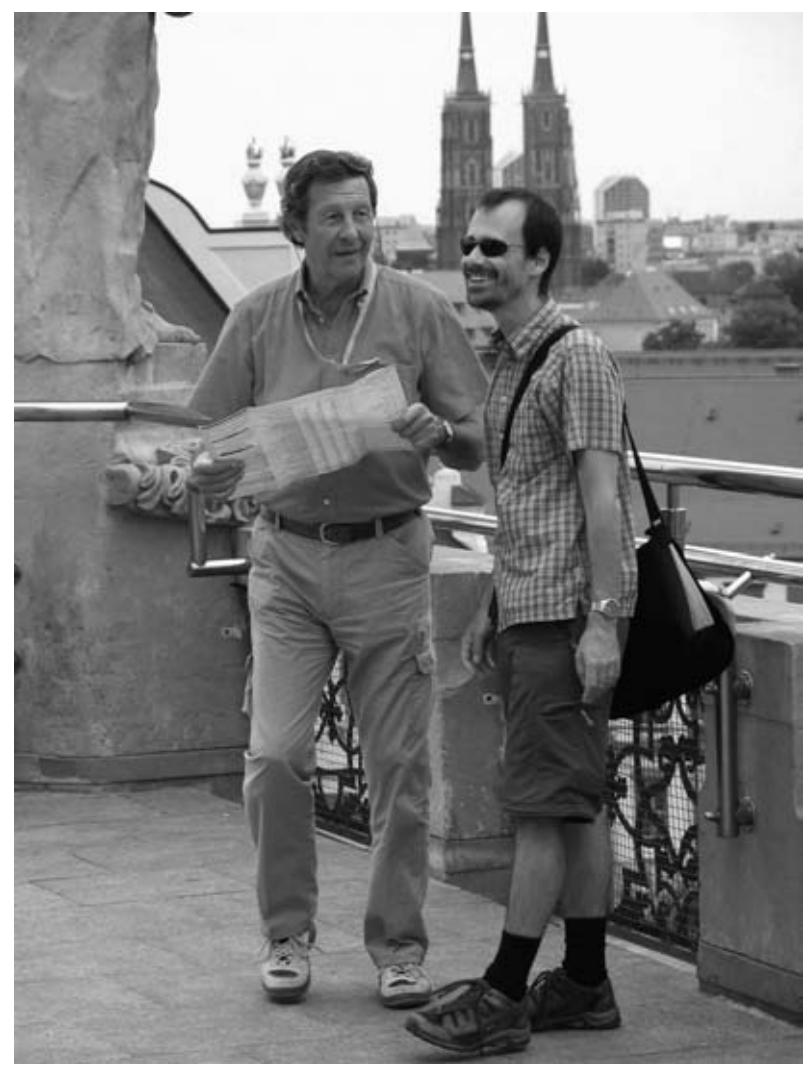

Nicolas Grevesse and Wolfgang Zima enjoy the city tour through Wrocław. 


\title{
Input from opacity data in computation of pulsation instability
}

\author{
J. Montalbán, and A. Miglio
}

Institut d'Astrophysique et Géophysique, Université de Liège, Allée du 6 Août, 4000 Liège, Belgium

\begin{abstract}
Several types of variable stars are found along the HR diagram whose pulsations are driven by the $\kappa$-mechanism. Given their nature, the precise $\left(T_{\text {eff }}-L\right)$ domain where these pulsators are located is highly dependent on the value of opacity and on its variation inside the star. We analyze the sensitivity of opacity driven pulsators of spectral-type $A$ and $B$ ( $\delta$ Scuti, $\beta$ Cephei and SPB stars) to the opacity tables (OP/OPAL) and to the chemical composition of the stellar matter. We also briefly discuss the effect of opacity on pulsators whose oscillations are not driven by the $\kappa$-mechanism, such as $\gamma$ Doradus and solar-like stars.
\end{abstract}

Individual Objects: 44 Tau

\section{Introduction}

Already in 1919, Eddington proposed that an increase of the absorption coefficient in some parts of the star at the moment of maximum compression could excite stellar pulsations. However, it was necessary to wait around forty years before Zhevakin (1959, and references therein) identified the region of the second ionization of $\mathrm{He}$ as responsible of pulsation excitation in cepheid variables. Baker \& Kippenhahn (1962) made the first numerical stability computations applied to realistic models of stellar envelope and confirmed that the increase of opacity in the region of second ionization of He leads to sufficient excitation to overcome the damping in the rest of the star, explaining in this way the pulsation of Cepheids. Since then, other pulsators have been observed whose excitation mechanism is linked to the variation of opacity with temperature and pressure, not only in the region of second ionization of helium, but also in the Fe-group opacity bump at $\log T \sim 5.3$ ( $\beta$ Cepheids and SPB's eg.

hboxDziembowski et al. 1993; sdB's, Kilkenny et al. 1997, Charpinet et al. 1996) and at $\log T \sim 6.25$ (Wolf-Rayet, Townsend \& MacDonald 2006).

The necessary conditions for the excitation of a pulsation mode of period $\tau$ by the $\kappa$-mechanism were described by Pamyatnykh (1999): $i$ ) the opacity perturbation in the high temperature phase must grow outward; ii) the amplitude of the pressure eigenfunction must be large and change slowly with radius in the driving region; iii) the thermal relaxation time $\left(4 \pi r^{3} \rho C_{p} T / L_{R}\right)$ in the driving region must be of the order or longer than the oscillation period $(\tau)$. The behavior of stellar opacity as a function of temperature and density is not only important in the framework of the first condition. Stellar opacity determines the density distribution of the equilibrium model and hence the oscillation frequencies. Moreover, the details of the stellar structure are in turn important in the balance between excitation and damping of oscillations. Finally, opacity of stellar plasma influences the location of convective regions, and hence may play a relevant role in the properties of oscillations. 

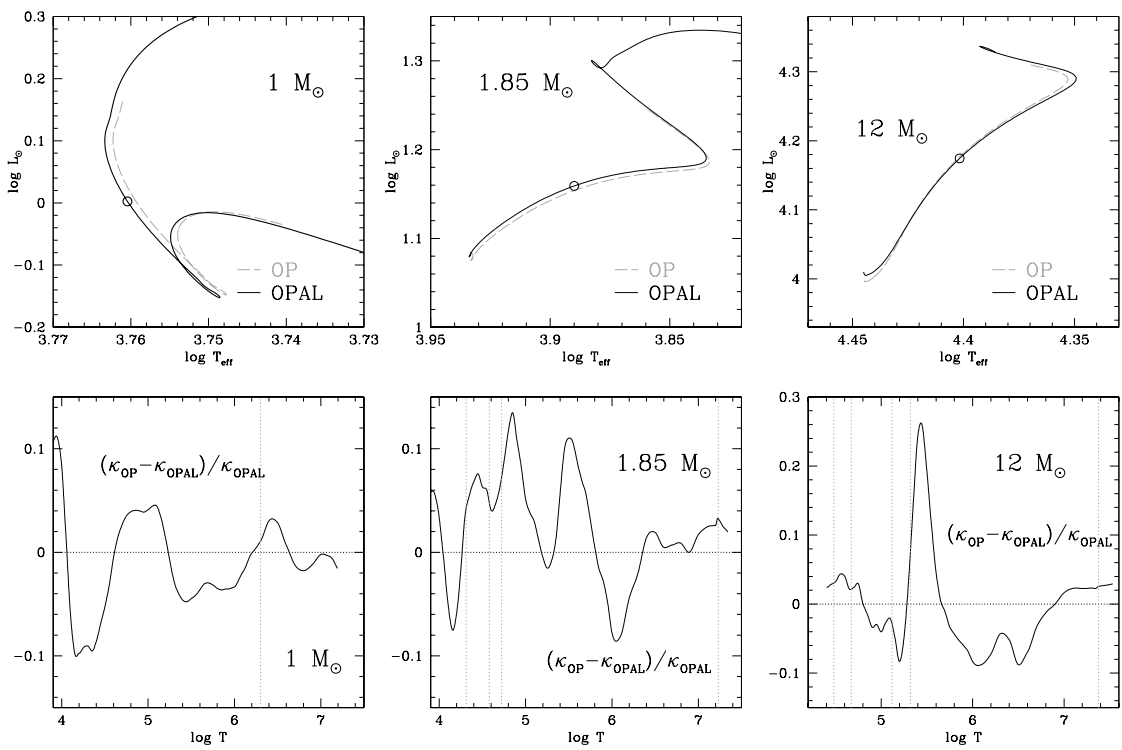

Figure 1: Upper panels: evolutionary tracks of $1 \mathrm{M}_{\odot}$ (left), $1.85 \mathrm{M}_{\odot}$ (center) and $12 \mathrm{M}_{\odot}$ (right) computed with OPAL (solid lines) and OP (dashed lines) opacity tables, same chemical composition $(X=0.70$, $Z=0.02)$ and treatment of convective transport. Lower panels: differences between OP and OPAL $\kappa_{R}$ through an OPAL model at the middle of the MS (mass fraction of hydrogen at the center $X_{\mathrm{c}}=0.35$ ). Vertical lines indicate the boundary of convective regions in those models.

The main ingredients in the computation of stellar opacities are two: atomic physics, and chemical composition of the stellar plasma. In this paper we examine the consequences of recent updates on the oscillation properties of different types of main sequence pulsators.

\section{Effect of opacity on stellar structure and evolution}

\section{Opacity tables}

The improvements included in the new OP (Opacity Project) opacity computations as well as a thorough comparison with OPAL (Iglesias \& Rogers 1996) opacity tables are described in Badnell et al. (2005). These changes result in an enhancement of Rosseland mean opacity, $\kappa_{\mathrm{R}}$, at high density and temperatures, and an increase of $18 \%$ of opacity in the Z-bump due to the new Fe atomic data. The new OP opacity tables are much closer to the OPAL ones than the previous release (Seaton et al. 1994). Some differences remain, however, at low temperatures $(\log T<5.5)$ : the OP Z-bump in $\kappa_{\mathrm{R}}$ presents a hot wing slightly larger than the OPAL one. This translates into an $\kappa_{\mathrm{R}}$ difference that can reach $30 \%$ at low densities. On the other hand, OP and OPAL agree at high density within $5-10 \%$.

This disagreement should affect differently models of typical solar-like, $\delta$-Scuti, and $\beta$ Cephei stars. In figure 1 we plot OP and OPAL evolutionary tracks for models with typical values of mass for these three kind of variables $\left(1.0,1.85\right.$, and $\left.12 \mathrm{M}_{\odot}\right)$. The 1.0 and $1.85 \mathrm{M}_{\odot}$ OP evolutionary tracks are cooler than the OPAL ones. Since all the stellar parameters in the models are the same, these results suggest a larger OP opacity with respect 

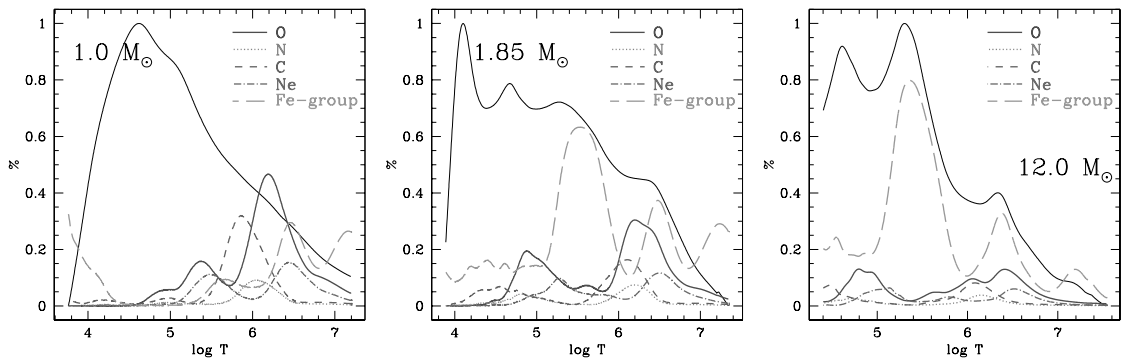

Figure 2: Profile of Rosseland mean opacity (thin solid line) for the same models as in figure 1. For each model we derived, by using the OP-sever facility ${ }^{1}$, the contribution to $\kappa_{\mathrm{R}}$ in percent coming from $\mathrm{O}$ (thick solid lines), N (dotted lines), C (short-dashed lines), Ne (dash-dotted lines) and from the elements of the iron group (long-dashed lines)

to the OPAL one. The differences across the stellar structure of main sequence models are shown in the lower panels of figure 1 . We emphasize a difference of $\sim 30 \%$ at $\log T \sim 5.3$ for the $12 \mathrm{M}_{\odot}$ model, that will have important consequences on the instability strip of B-type pulsators, and also that OP opacities are systematically $10 \%$ lower than the OPAL ones in the region around $\log T \sim 6$.

\section{Solar mixture}

The re-analysis of solar spectrum by Asplund et al. (2005) (hereafter AGS05) including NLTE effect as well as tridimensional model atmosphere computations, has led to a significant decrease of $\mathrm{C}, \mathrm{N}, \mathrm{O}$ and $\mathrm{Ne}$ solar abundances and as a consequence, to a solar metallicity $\left((Z / X)_{\odot}\right) 30 \%$ smaller than the value provided by the "standard" GN93 mixture (Grevesse \& Noels 1993). The abundance of iron $(\log N(\mathrm{Fe}) / N(\mathrm{H}))$ has not been affected by the new analysis, which means an iron mass fraction in AGS05 mixture $25 \%$ larger in than in GN93 one for a given $Z$. It is worth noting that while metallicity is mainly determined by the abundance of $\mathrm{C}, \mathrm{N}$ and $\mathrm{O}$, the value of the Rosseland mean opacity can be strongly affected by other less abundant elements. The contribution to opacity from each element in $Z$ depends on density and temperature, hence the effects of solar mixture changes on stellar pulsation properties are expected to depend on spectral type. Figure 2 shows the variation of $\kappa_{\mathrm{R}}$ with temperature through the structure of MS stellar models with masses of 1.0, 1.85 and $12.0 \mathrm{M}_{\odot}$, and for each of them, the contribution of $\mathrm{C}, \mathrm{N}, \mathrm{O}, \mathrm{Ne}$ and Fe-group elements $(\mathrm{Fe}$, $\mathrm{Ni}, \mathrm{Co}, \mathrm{Mn}$ ) to $\kappa_{\mathrm{R}}$. For $1.85 \mathrm{M}_{\odot}$ (typical $\delta$ Scuti star), $\kappa_{\mathrm{R}}$ (thin solid line) clearly shows the peaks due to $\mathrm{H}$, and $\mathrm{He}$ ionization $(\log T \sim 4.1)$, to $\mathrm{He}^{+}$ionization $(\log T \sim 4.6)$, and to the so-called Z-bump of opacity at $\log T \sim 5.3)$. The opacity peak due to $\mathrm{He}^{+}$ionization is also evident in $\kappa_{\mathrm{R}}$ curve for $12.0 \mathrm{M}_{\odot}$ model, nevertheless, for this model the main contribution to opacity comes from Fe-group elements. We also notice that the contribution of CNO to $\kappa_{\mathrm{R}}$ decreases as stellar mass increases.

The total effect of new solar mixture on the value of $\kappa_{R}$ is shown in figure 3 . It should be noticed that for a given value of metal mass fraction $Z$, AGS05 mixture leads to a larger $\kappa_{\mathrm{R}}$ than GN93 one. Nevertheless, it should be kept in mind that the new $Z_{\odot}$ is only $70 \%$ of the old one. The hollow at $\log T \sim 6.2$ in $\delta \kappa_{\mathrm{R}}$ for 1.0 and $1.85 \mathrm{M}_{\odot}$ (left and central panels in Fig.3) is the signature of the lower oxygen-abundance in AGS05 mixture. While the $30 \%$ decrease of $\mathrm{C}, \mathrm{N}, \mathrm{O}$ abundances increases the discrepancies between the standard

\footnotetext{
${ }^{1}$ http://opacities.osc.edu/rmos.shtml
} 

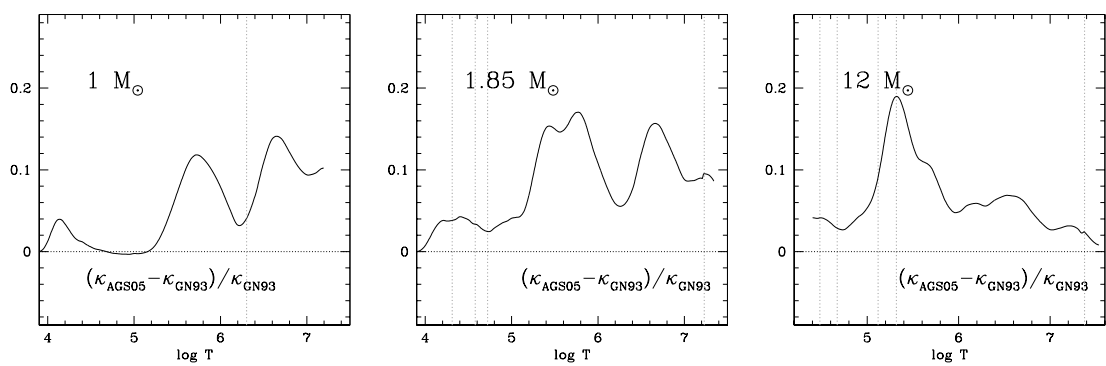

Figure 3: Relative differences of $\kappa_{\mathrm{R}}$ values provided by GN93 and AGS05 metal mixtures at fixed $Z=0.02$, through the structure of the GN93 models. The models considered in the left, central and right panel are the same as in figures 1 and 2. Vertical lines corresponds to the boundaries of convective regions.

solar model and helioseismology, AGS05 values are in a better agreement with spectroscopic measurements in B-type stars (Morel et al. 2006). Moreover, since Fe-group elements are the main contributors to opacity for these stars, the increase of the iron mass fraction by $25 \%$ in the AGS05 mixture will favorably affect the excitation of $\beta$ Cep and SPB pulsation modes in early-type stars.

\section{Opacity driven pulsators}

\section{B-type pulsators}

$\beta$ Cep and Slow Pulsating B (SPB) stars (spectral type B0.5-B3 the former, and B3-B8 the latter) are MS pulsators excited by the $\kappa$-mechanism due to the Fe-group opacity bump at $T \sim 2 \times 10^{5} \mathrm{~K}$ (e.g. Dziembowski et al. 1993). OP opacity values in the Z-bump are larger than OPAL ones by almost $30 \%$ for a typical $\beta$ Cep, moreover the increase by $25 \%$ of iron mass fraction for a given $Z$ implies a higher and larger peak in $\kappa_{\mathrm{R}}$ at $T \sim 2 \times 10^{5} \mathrm{~K}$. The combined effect of new opacity and solar mixture on the SPB and $\beta$ Cephei instability strip has been examined by Miglio et al. (2007a,b) and Pamyatnykh (2007). Their results can be summarized as follows: while the different profile of $\kappa$ in OP and OPAL computations modifies the blue border of the instability strip, a larger Fe-mass fraction in the metal mixture provides slightly wider instability bands, and this effect increases as the metallicity decreases. Thus, the number of $\beta$ Cep pulsators expected with AGS05 is more than three times larger than with GN93, and the SPB instability strip resulting from OP calculations is $3000 \mathrm{~K}$ larger than with OPAL ones.

As a consequence, the number of expected hybrid $\beta$ Cep-SPB objects is also larger for OP models. The Fe-mass fraction enhancement in the AGS05 mixture, compared with GN93, has the main effect of extending towards higher overtones the range of excited frequencies. While for $Z=0.01$ the increase of B-type pulsators resulting from the updates of physics in the models is remarkable, for $Z=0.005$ SPB-type modes are excited when considering OP with AGS05, but none of the different OP/OPAL and GN93/AGS05 evolutionary tracks for masses up to $18 M_{\odot}$ predicts $\beta$ Cep pulsators.

The updates in basic physics lead hence to a decrease in the disagreement between theory and observations. In fact, the presence of B-type pulsators in low-metallicity environments (Kolaczkowski et al 2006, Karoff et al. 2008, Diago et al. 2008), as well as that of hybrid $\beta$ Cep-SPB pulsators (Handler et al. 2006; Jerzykiewicz et al 2005) cannot be explained in the context of OPAL opacities and GN93 mixture. Nevertheless, some difficulties re- 


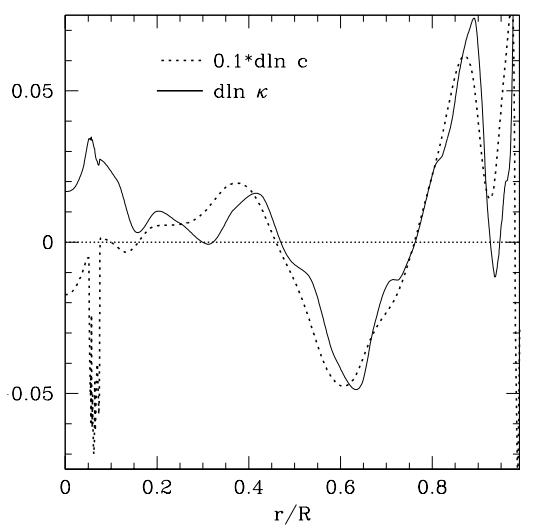

Figure 4: Relative differences between OPAL and $\mathrm{OP} \kappa_{\mathrm{R}}$ trough the structure of an OPAL model with $M=1.875 \mathrm{M}_{\odot} Z=0.02, X=0.70$ and central mass fraction of hydrogen $X_{c}=0.4$. Dotted-line represents the relative difference of sound speed at fixed mass for the two models with the same parameters but different opacity tables (OP et OPAL).

main, for instance, on how to explain the presence of $\beta$ Cep pulsators observed in the Small Magellanic Cloud (Kolaczkowski et al 2006), and the pulsation spectrum detected in $\nu$ Eri (Dziembowski \& Pamyatnykh 2008).

The understanding of pulsations in subluminous $B$ stars $(\mathrm{sdB})$ has also benefited from the updated opacity values (Jeffery \& Saio 2006), showing that B-type pulsators can be considered as a "critical test for stellar opacity".

\section{$\delta$ Scuti stars: 44 Tau}

$\delta$ Scuti stars (A-F spectral type) are located in the low luminosity end of the classical instability strip. They pulsate in low order $\mathrm{p}$ and mixed g-p modes excited by the $\kappa$-mechanism in the second ionization region of helium, at $\log T \sim 4.6$. In a recent paper, Lenz et al. (2008) have analyzed the effects of solar mixture and opacity tables in the seismic modeling of $44 \mathrm{Tau}$, a slowly rotating $\delta$ Sct variable that shows radial and nonradial pulsation modes (Antoci et al. 2007; Zima et al. 2007). The values of periods of fundamental $\left(\Pi_{0}\right)$ and first overtone $\left(\Pi_{1}\right)$ radial modes together with the low rotational velocity $\left(v \sin i<5 \mathrm{~km} \mathrm{~s}^{-1}\right)$ allow Lenz et al. (2008) to use the Petersen diagram $\left(\Pi_{1} / \Pi_{0} v s \log \Pi_{0}\right)$ to derive the fundamental parameters of $44 \mathrm{Tau}$ and to analyze their dependence on the basic physics. Comparison between OP and OPAL opacities through a typical $\delta$ Scuti structure (central panel of fig. 1 ) does not show a significant difference (lower than $5 \%$ ) in the driving region. Nevertheless, the authors found that for the case of 44 Tau the OPAL opacities are preferable to the OP ones, since it was not possible to fit the radial modes of $44 \mathrm{Tau}$ at the same time than its location in the HR diagram by using OP models.

The Petersen diagrams (Petersen \& Jorgensen 1972) are known to be very sensitive to the metallicity, and hence to opacity. To clarify the results obtained by Lenz et al. (2008) we compared OPAL and OP stellar models with the same mass $\left(\mathrm{M}=1.875 \mathrm{M}_{\odot}\right.$, such as derived by Lenz et al. 2008) and at the same evolutionary stage $\left(X_{c}=0.4\right)$. Their location in the $\mathrm{HR}$ diagrams is quite close, their radii differ by $0.05 \%$, and hence their fundamental period, $\Pi_{0}$, by $0.16 \%\left(\log \Pi_{0}=-0.8380\right.$ instead of -0.8387$)$. The effect on the period ratio is however larger, going from 0.7697 for the OPAL model to 0.7722 for the OP one. This difference is an indication of a lower OP opacity with respect to OPAL values. In figure 1 , we see that OP is lower than OPAL by a $10 \%$ in the region around $\log T \sim 6.05$.

As explained by Petersen \& Jorgensen (1972), the period ratio $\left(\Pi_{1} / \Pi_{0}\right)$ mainly depends on the effective politropic index $(n=d \ln P / d \ln T-1)$ of plasma in a region around 

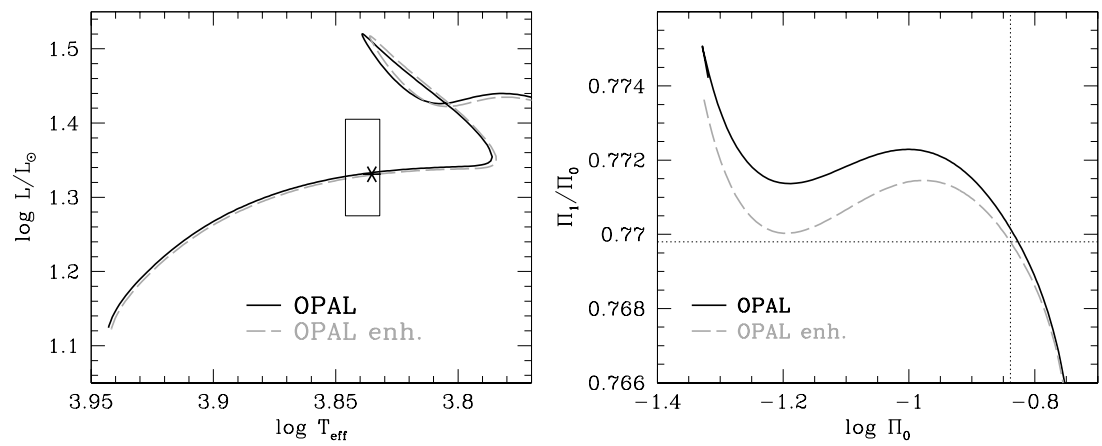

Figure 5: Left panel: evolutionary tracks for $M=1.9 \mathrm{M}_{\odot}, Z=0.02, X=0.7, \alpha_{\mathrm{OV}}=0.3$ computed with OPAL opacity tables (solid line) and with OPAL enhanced by $5 \%$ in the region around $\log T \sim 6.05$ (dashed line). The square corresponds to the observational error box (Lenz et al. 2008), and the star represent the model fitting $\Pi_{0}$ and the period ratio $\left(\Pi_{1} / \Pi_{0}\right)$. Right panel: Petersen diagram corresponding to the evolutionary tracks in left panel.

$x=r / R=0.7$, much deeper than the second ionization zone of helium. For their stellar envelope models, this region corresponds to $\log T$ between 5.5 and 6.2. Figure 4 shows that the largest difference between OP and OPAL opacity values is found close to $x=0.7$. Different $\kappa_{\mathrm{R}}$ involves a different sound speed inside the star and hence different pulsation periods. A lower opacity implies larger values of period ratio. Hence, fitting with OP models the observational $\Pi_{0}$ and $\Pi_{1} / \Pi_{0}$ of 44 Tau required Lenz et al. (2008) to decrease by $10 \%$ the mass of the model, and therefore the effective temperature and luminosity of the model were not compatible with the observational error box.

Given the sensitivity of the period ratio to the opacity at $\log T \sim 6.05$ we wonder how the stellar parameters derived by using the Petersen's diagram depend on the uncertainty affecting the opacity values. We found that by increasing the OPAL opacities by at maximum a $5 \%$ around $\log T \sim 6.25$, a MS model with $M=1.9 \mathrm{M}_{\odot} X=0.70, Z=0.02, X_{\mathrm{c}}=$ 0.195 and an overshooting parameter $\alpha=0.3$ satisfies the observational constrains (HR and Petersen diagrams locations) as shown in figure 5. This differs from the result found in Lenz et al. (2008), where only post-MS models were able to fit observations.

\section{Other pulsators}

\section{$\gamma$ Doradus pulsators}

$\gamma$ Doradus variables are F-type stars oscillating with high-order g-modes (Kaye et al. 1999), and the excitation mechanism has been identified by Guzik et al. (2000) as being due to the convective blocking of radiative flux. The location of the convective zone (and hence potentially stellar opacity) plays then a fundamental role in these pulsators. Computations considering the interaction pulsation-convection by Dupret et al. (2005) confirm the first results by Guzik obtained in the frozen convection approximation and shows that there is also a small contribution of the $\kappa$-mechanism coming from the Z-bump of opacity at $\log T \sim 5.3$, close to the bottom of the convective envelope in $\gamma$ Dor stars. Despite all that, changing opacity tables and the solar mixture do not significantly affect neither the instability strip nor the oscillation spectrum. Nevertheless, these objects are still not well-known, and some surprises could rise in the future. 
The Sun

Oxygen is the main contributor to $\kappa_{\mathrm{R}}$ at the bottom of the solar convective envelope ( $\log T \sim 6.3$ ), followed by iron and neon. The decrease by $30 \%$ of solar metallicity resulting from Asplund et al. (2005) analysis has hence led to drop by $20 \%$ the opacity at the bottom of convective zone and to ruin the good agreement between the standard solar model and helioseismology (see Basu \& Antia 2008 and references therein). The updated OP opacities imply only a raise of opacity at the bottom of the convective zone at maximum of $5 \%$, too small to solve the problem of the helioseismic model. A large variety of suggestions have been proposed to recover the lost opacity and hence the agreement with helioseismic results, however none up to now (Basu \& Antia 2008 for review) has provided satisfactory results, and the problem of the solar model remains. We would like, nevertheless, to recall that the good agreement between the Solar Standard Model (Christensen-Dalsgaard et al. 1996) and the seismic one was also damaged (even if the effect was small compared with the actual solar problem) when the updated version of OPAL opacity tables (Iglesias \& Rogers 1996) led to a decrease of opacity of $5 \%$ at the bottom of solar convective region $\log T \sim 6.3$. The so-called S-model was in fact computed with the first delivery of OPAL opacity tables (Rogers \& Iglesias 1992).

\section{Conclusions}

In the nineties, the OPAL opacity tables signified a revolution in stellar physics. Since then, the progress in observational techniques and numerical computations have highlighted new discrepancies between theory and observations. In spite of recent improvements some problems persist, for instance: i) the presence of $\beta$ Cep pulsators in the SMC; $i$ i) the excitation of high and low frequencies in B-type pulsators such as $\nu$ Eri (Dziembowski \& Pamyatnykh 2008); iii) discrepancy between solar model and helioseismology; $i v$ ) the remaining $15 \%$ disagreement between evolution and pulsation masses of cepheids variables (Keller 2008 and references therein). Some of these discrepancies could be reduced by increasing (yes, again...) $\kappa_{\mathrm{R}}$ in limited domains of temperature, in particular at $\log T>6$ (see also Zdrawkov \& Pamyatnykh 2008).

In spite of recent updates, some differences between OP and OPAL remain, mainly due to the different equation of state used in those approaches. The differences may seem small ( $\sim 10 \%$ ), but we showed here that an enhancement of $5 \%$ at $\log T \sim 6$ may be able to modify the results of a seismic analysis. Both opacity compilations, OP and OPAL, were computed assuming reasonable approximations for the then current accuracy of observations. So, perhaps there is some room to increase stellar opacity. In fact, not all the elements are considered with the same degree of accuracy, and heavy and low abundance elements have not been taken in consideration since their contributions to $\kappa_{\mathrm{R}}$ were estimated to be small (Iglesias et al. 1995) for the solar model, and the computation very time-consuming.

Stellar pulsation theory has come out to be a powerful tool to probe different aspects of the stellar physics. Today, this suggests that perhaps it is time to come back to review the basic input physics in stellar modeling, in particular stellar opacity computations.

Acknowledgments. JM and AM acknowledge financial support from the European Helioand Asteroseismology Network HELAS, from the Prodex-ESA Contract Prodex 8 COROT (C90199) and from FNRS. 


\section{References}

Antoci, V., Breger, M., Rodler, F., et al. 2007, A\&A, 463, 225

Asplund, M., Grevesse, N., \& Sauval, A. J. 2005, ASP Conference Series, 336, 25

Badnell, N. R., Bautista, M. A., Butler, K.,et al. 2005, PhR, 360, 458

Baker, N., \& Kippenhahn, R. 1962, Z.A., 54, 114

Charpinet, S., Fontaine, G., Brassard, P., \& Dorman, B. 1996, ApJ, 471, L103

Christensen-Dalsgaard, J., Dappen, W., Ajukov, S. V., et al. 1996, Science, 272, 1286

Diago, P. D., Gutiérrez-Soto, J., Fabregat, J., \& Martayan, C. 2008, A\&A, 480, 179

Dupret, M.-A., Grigahcène, A., Garrido, R., et al. 2005, A\&A, 435, 927

Dziembowski, W. A., \& Pamyatnykh, A. A. 2008, MNRAS, 385, 2061

Dziembowski, W. A., Moskalik, P., \& Pamyatnykh, A. A. 1993, MNRAS, 265, 588

Grevesse, N., \& Noels, A. 1993, in Hauck B. Paltani S. R. D., ed., La formation des éléments chimiques, AVCP La composition chimique du soleil

Guzik, J. A., Kaye, A. B., Bradley, P. A., et al. 2000, ApJ, 542, L57

Handler, G., Jerzykiewicz, M., Rodrguez, E., et al. 2006, MNRAS, 365, 327

Iglesias, C. A., Wilson, B. G., Rogers, F. J., et al. 1995, ApJ, 445, 855

Iglesias, C. A., \& Rogers, F. J. 1996, ApJ, 464, 943

Karoff, C., Arentoft, T., Glowienka, L., et al. 2008, MNRAS, 386, 1085

Keller, S. C. 2008, ApJ, 677, 483

Jeffery, C. S., \& Saio, H. 2006, MNRAS, 372, L48

Jerzykiewicz, M., Handler, G., Shobbrook, R. R., et al. 2005, MNRAS, 360, 619

Kaye, A. B., Handler, G., Krisciunas, K., et al. 1999, PASP, 111, 840

Kilkenny, D., Koen, C., O'Donoghue, D., \& Stobie, R. S. 1997, MNRAS, 285, 640

Kołaczkowski, Z., Pigulski, A., Soszyński, I., et al. 2006, MemSAlt, 77, 336

Lenz, P., Pamyatnykh, A. A., Breger, M., \& Antoci, V. 2008, A\&A, 478, 855

Miglio, A., Montalbán, J., \& Dupret, M.-A. 2007a, MNRAS, 375, L21

Miglio, A., Montalbán, J., \& Dupret, M.-A. 2007b, CoAst, 151, 48

Morel, T., Butler, K., Aerts, C., et al. 2006, A\&A, 457, 651

Pamyatnykh, A. A. 1999, Acta Astronomica, 49, 119

Pamyatnykh, A. A. 2007, CoAst, 150, 207

Petersen, J. O., \& Jørgensen, H. E. 1972, A\&A, 17, 367

Rogers, F. J., \& Iglesias, C. A. 1992, ApJS, 79, 507

Seaton, M. J., Yan, Y., Mihalas, D., \& Pradhan, A. K. 1994, MNRAS, 266, 805

Townsend, R. H. D., \& MacDonald, J. 2006, MNRAS, 368, L57

Zhevakin, S. A. 1959, Soviet Astronomy, 3, 913

Zima, W., Lehmann, H., Stütz, C., et al. 2007, A\&A, 471, 237

Zdrawkov \& Pamyatnykh 2008, CoAst, 157, 385 


\title{
Radiative levitation and opacity driving: The potential of hot subdwarf pulsators for testing diffusion and other competing processes in stars
}

\author{
S. Charpinet ${ }^{1}$, G. Fontaine ${ }^{2}$, P. Brassard ${ }^{2}$, and P. Chayer ${ }^{3}$ \\ ${ }^{1}$ LATT, Université de Toulouse, CNRS, 14 av. E. Belin, 31400 Toulouse, France \\ 2 Département de Physique, Université de Montréal, \\ CP 6128, Succursale Centre-Ville, Montréal, QC H3C 3J7, Canada \\ ${ }^{3}$ Space Telescope Science Institute, 3700 San Martin Drive, Baltimore, MD 21218, USA
}

\begin{abstract}
The hot and compact subdwarf ( $\mathrm{sdB}$ and sdO) stars harbor three classes of nonradial pulsators: the rapid p-mode EC 14026-type sdB pulsators discovered in 1997, the slow g-mode sdB pulsators known as the $\mathrm{V} 1093 \mathrm{Her}$ variables found in 2003, and the rapid sdO pulsators identified in 2006. The oscillations in these stars are singular in that the driving mechanism - a classical kappa effect triggered by the Z-bump, a region where partial ionisation of heavy elements from the iron group significantly increases the gas opacity - is truly effective only through local abundance enhancements of such elements (in particular of iron itself) caused by microscopic diffusion. They are the only pulsating stars, among those currently known across the HR diagram, that show oscillations so tightly linked to these diffusive phenomena. In this paper, we present a short review of our present understanding of the various components that drive pulsations in subdwarf stars and we outline the remarkable potential of these stars as laboratories where diffusion and other competing processes may be tested.
\end{abstract}

Individual Objects: $\quad$ PG 1325+101, Feige 48

\section{Introduction}

The B subdwarf (sdB) stars are hot and compact objects identified with the so-called Extreme Horizontal Branch (EHB) stars burning helium in their core. Their atmospheric parameters are found in the ranges $20,000 \mathrm{~K} \leq T_{\text {eff }} \leq 40,000 \mathrm{~K}$ and $5.0 \leq \log g \leq 6.2$. The hot $\mathrm{O}$ subdwarf ( $\mathrm{sdO}$ ) stars presumably result from various evolutionary histories, a fraction of them being most likely descendants of sdB stars that have reached post-EHB evolution after core helium exhaustion. The atmospheric parameters of $\mathrm{sdO}$ stars are spreaded over wide ranges covering $40,000-100,000 \mathrm{~K}$ for $T_{\text {eff }}$ and $4.0-6.5$ for $\log g$.

Three groups of low-amplitude multiperiodic nonradial pulsators have been identified among hot subdwarf stars. These are the short-period B subdwarf pulsators (the V361 Hya or EC 14026 stars) found by Kilkenny et al. (1997) oscillating in low-order p-modes with periods in the range $80-600 \mathrm{~s}$, the long-period sdB pulsators (the V1093 Her, PG1716+426, or "Betsy" stars) discovered by Green et al. (2003) showing mid-order g-modes with periods between 2000 and 9000 seconds, and the short period sdO pulsators, whose unique known member thus far is the star SDSS J160043.6+074802.9 (Wouldt et al. 2006) having periods in the $60-120 \mathrm{~s}$ range. Each of these classes harbor a strong potential for detailed asteroseismic studies. So far, this has been exploited mostly for the short-period sdB pulsators (see, e.g., the recent review of Fontaine et al. 2008a). 

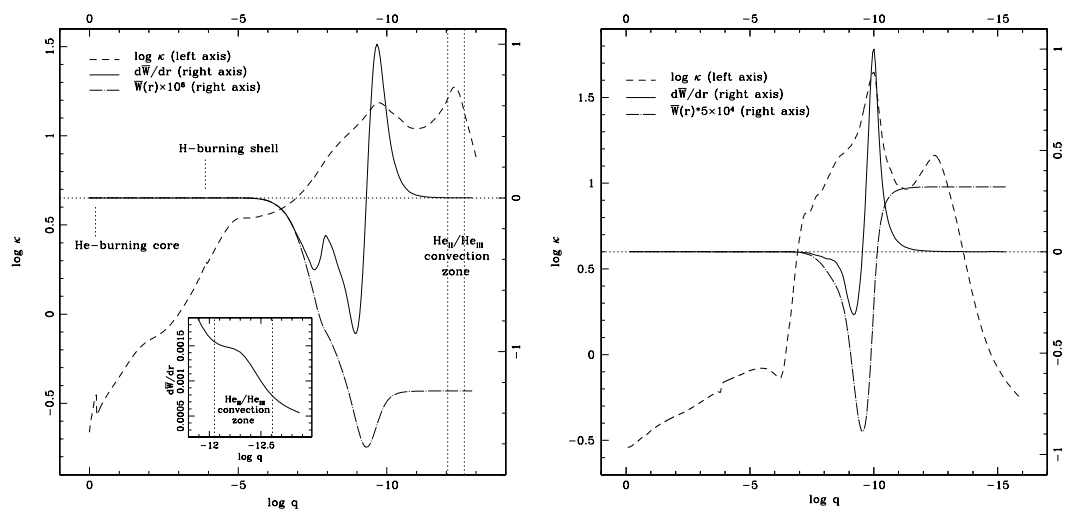

Figure 1: Rosseland mean opacity (dashed line), integrand of the work integral (solid line), and running work integral (dotted-dashed line) as functions of the fractional mass depth $\log q=\log (1-m(r) / M)$. Left panel shows a representative (globally damped) $p$-mode in a sdB model with a uniform solar metallicity $(Z=0.02)$ in the envelope. Right panel shows a representative (globally driven) $p$-mode in a sdB model with a nonuniform iron profile in the envelope from equilibrium between gravitational settling and radiative levitation. Local mode driving (regions where $d W / d r>0$ ) is clearly associated with the Z-bump region in the mean Rosseland opacity and is strongly enhanced (to the point of destabilizing modes) in the models incorporating radiative levitation.

Hot subdwarfs also present an interesting - yet unexploited - potential link to the specificities of the driving mechanism. In the present paper, we briefly recall the nature of this mechanism and the physical processes involved. Then we outline new opportunities that such pulsators may indeed provide for studying diffusive and mixing processes in stars.

\section{Driving pulsations in hot subdwarf stars}

Among oscillating stars, hot pulsating subdwarfs are singular in that radiative levitation of heavy elements (especially iron) is expected to play a central role in explaining the very existence of the pulsations themselves. The intimate relationship between the envelope metal content and the pulsation driving process was first established by Charpinet et al. (1996) for the short-period B subdwarf pulsators. It was demonstrated that $p$-mode instabilities can occur in sdB stellar models through the action of a classical $\kappa$-mechanism involving mainly partial ionization of the M-shell electrons of iron. The combined effect of millions of spectral lines from the iron M-shell ions results in a sharp peak, often called the Z-bump, in the Rosseland opacity profile. It was also shown that a solar content of metals in the envelope is unable, however, to produce enough driving to globally destabilize modes, thus implying that an additional process must be involved to boost the $\kappa$-effect (see left panel of Figure 1 ).

This process is most likely radiative levitation, which is expected to act efficiently in the stable radiative envelopes of hot subdwarf stars. In competition with other processes, such as gravitational settling and possibly weak stellar winds, radiative levitation is believed to be responsible for the notorious atmospheric abundance anomalies observed in sdB stars (see, Blanchette et al. 2008 and references therein; left panel of Figure 2). It is also expected to produce reservoirs of levitating elements below the photosphere, although these reservoirs cannot be observed directly as these optically thick layers remain hidden to spectroscopic studies. In particular, iron tends to accumulate nonuniformely at relatively large extrasolar abundances 

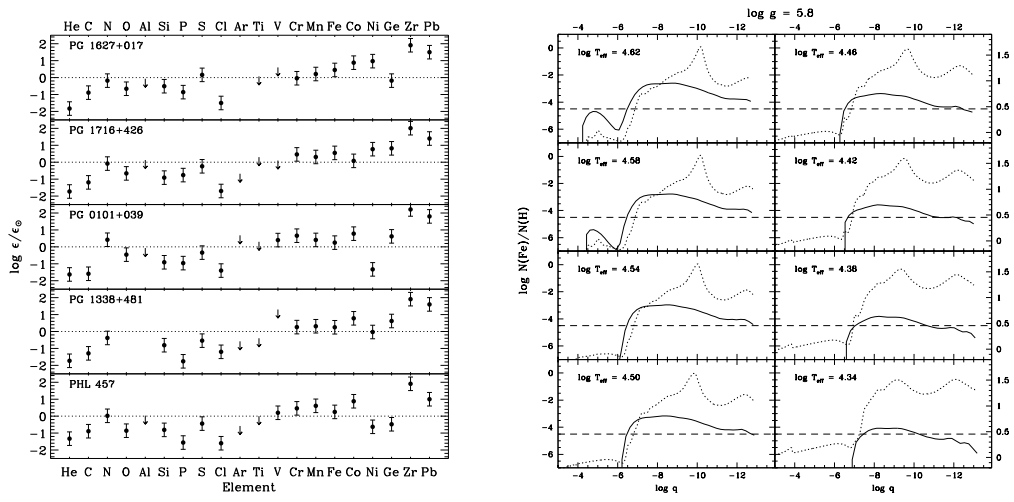

Figure 2: Left panel (Figure from Blanchette et al. 2008) shows typical examples of anomalous element distributions in the atmosphere of $\mathrm{sdB}$ stars. The values given are relative to solar content. For various effective temperatures (at fixed $\log g$ ), right panel illustrates representative iron distributions (solid curves) and Rosseland opacity profiles (dotted curves) in sdB stars assuming diffusive equilibrium between radiative levitation and gravitational settling. The horizontal dashed line indicates the solar amount of iron.

in the stellar envelope (right panel of Figure 2) with the effect of powering up the driving mechanism and producing efficient excitation for pulsation modes (Charpinet et al. 1997; see right panel of Figure 1 ). Remarkably, the same $\kappa$-effect involving radiative levitation is likely responsible for the driving of $g$-modes in the long period sdB pulsators (Fontaine et al. 2003), and for the driving of very short period $p$-modes in the newly discovered class of hot pulsating sdO stars (Fontaine et al. 2008b, and Fontaine \& Brassard 2008).

The identification of the main constituents producing the driving of pulsations in sdB stars inspired the development of new stellar models dealing with the important role of radiative levitation (the so-called "second generation models"; Charpinet et al. 1997). These models are constructed around the assumption that a state of diffusive equilibrium is reached between radiative levitation and gravitational settling (thus disregarding, at this stage, other potentially competing processes), leading to stable nonuniform abundance profiles for chemical species in the stellar envelope (right panel of Figure 2). It is indeed expected, from time-dependent diffusion calculations, that this state is reached over timescales much shorter than the typical lifetime of sdB stars, which is $\sim 1.5 \times 10^{8} \mathrm{yr}$ (see Fontaine et al. 2006a and Figure 3). These calculations demonstrate that mode instabilities appear after $\sim 10^{5} \mathrm{yr}$ (transition between red and green curves in the color version of Figure 3) and are fully set, i.e., the whole range of modes is driven, after $\sim 10^{6} \mathrm{yr}$, only. It then takes $\sim 10^{7} \mathrm{yr}$ for the levitating reservoir of iron to be fully in equilibrium in the stellar envelope. Another simplification introduced in the second generation models is that only iron is allowed to levitate in a pure hydrogen envelope, a choice justified by the facts that iron is the dominant contributor to the Z-bump opacity, and that helium is well-known to be very underabundant in the envelopes/atmospheres of $\mathrm{sdB}$ stars (a situation presumably caused by rapid sedimentation). The real picture might be more complicated, however, as pointed out recently by Jeffery \& Saio (2006a, 2006b, 2007) who argued that nickel may also contribute for a significant fraction to the stellar gas opacity in the Z-bump region. They also noticed important differences between the OPAL opacities and the most recent OP opacities having a direct impact on the properties of the pulsation driving mechanism. In particular, they found indications that models using the OP opacities may better reproduce the position of the instability region for the long period g-mode sdB pulsators, although they did not include radiative levitation in their calculations. 


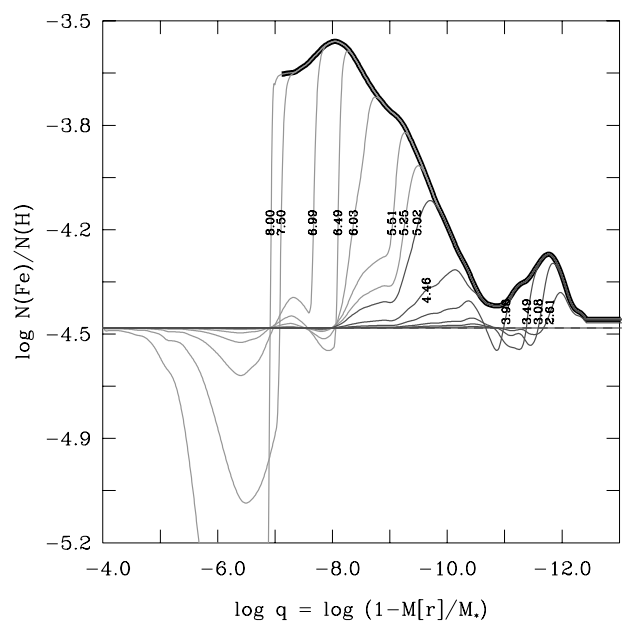

Figure 3: Evolving iron abundance distribution in the envelope of a typical sdB model with $M=0.5 M_{\odot}$, $T_{\text {eff }}=30,000 \mathrm{~K}$, and $\log g=5.5$. The thick solid curve represents the equilibrium Fe profile. Numbers associated with each thin solid curves indicate the time - in logarithmic scale - passed since the Zero Age Extreme Horizontal Branch (where the iron abundance distribution is assumed uniform and solar). Red curves (in the color version) indicate models that show no mode instabilities while green curves indicate models that develop pulsation mode instabilities.

Despite potential shortcomings and uncertainties alluded to above, second generation models have constituted a substantial improvement over stellar structures based on chemically uniform abundances for the study of hot subdwarf pulsators. For instance, the class properties of pulsating sdB stars are globally well-reproduced with these models and they have been successfully used for detailed asteroseismic studies of several EC 14026 stars (Charpinet et al. 2007). However, model improvements are still necessary to tackle some of the pending issues in the field. Among these, understanding why only a minority of stars that fall within the empirical EC 14026 instability domain actually pulsate is one of the current challenges (although a solution might reside in the presence of weak stellar winds in that case; Fontaine et al. 2006b). Another issue concerns the actual extent of the observed instability strip for the EC 14026 stars, which is narrower than the predicted one, and which can be correlated with the fact that the observed period ranges in individual stars are also usually narrower than predicted. All of this is plausibly linked to approximations and factors that control and modulate the efficiency of the driving mechanism, themselves resulting from the interplay of various competing processes not included in the current second generation models.

\section{The main factor controlling the pulsation engine}

Developing accurate descriptions of competing diffusive and mixing processes in stars is among the major challenges that modern stellar astrophysics will have to face (see the excellent review of Dupret (2008) in these proceedings). In stars whose role is significant, these processes will shape the chemical stratification in the interior and affect the structural and evolutionary 

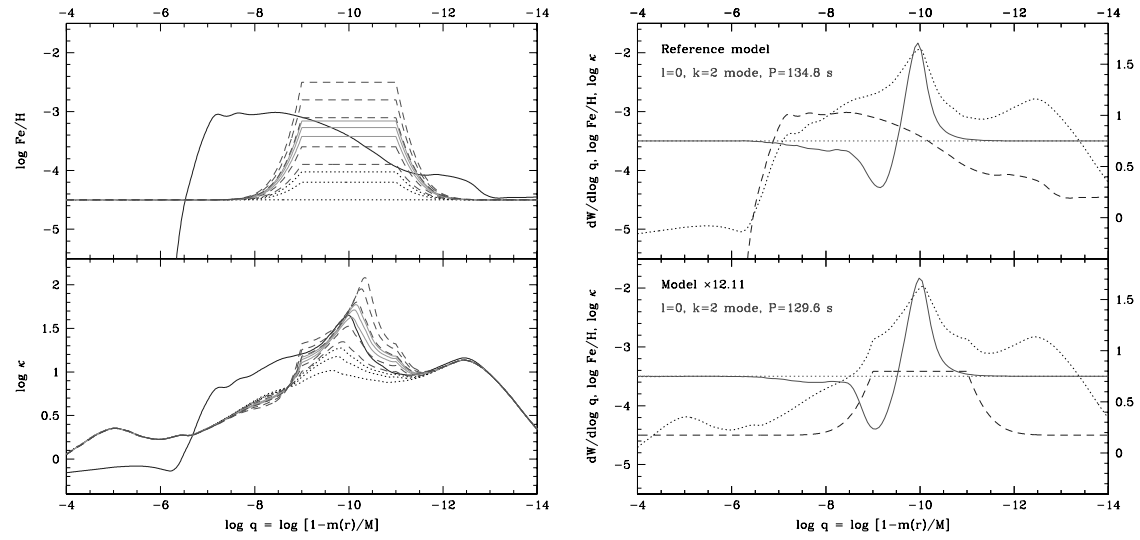

Figure 4: Left plot, upper panel: Iron content, log $\mathrm{Fe} / \mathrm{H}$ (in number), as a function of fractional mass depth, $\log q$, for a series of models. The blue solid curve shows the iron profile predicted from equilibrium between gravitational settling and radiative levitation (the reference model). Other profiles are from models constructed to produce an enrichment of iron only in the Z-bump region (and solar everywhere else). From bottom to top, the black dotted-lined step profiles represent models with local enrichments of $\times 1, \times 2$, $\times 3$ the solar value $(\log \mathrm{Fe} / \mathrm{H}=-4.5)$ where no mode driving is found. The red dashed-lined step profiles are models with $\mathrm{Fe}$ local enrichments of $\times 4, \times 8, \times 25, \times 50, \times 100$ the solar value showing $p$-modes instabilities. The green solid-line profiles are models with local Fe enrichments of $\times 12, \times 17$, and $\times 22$ showing a range of excited $p$-modes similar to the reference model. Left plot, lower panel: Logarithm of the Rosseland opacity, $\log \kappa$, as a function $\log q$ for the same series of models. Color and curve conventions are the same as above. Right plot: Driving and damping structure for a representative $\ell=0, k=2$ globally excited $p$-mode from the reference model (upper panel) and from its corresponding Z-bump enhanced model $(\times 12.11$ the solar value, lower panel). The red solid curve shows the integrand of the work integral of the mode as a function of $\log q$. The blue dashed curve is the iron abundance profile, to be read on the LHS axis. The black dotted curve gives the run of the Rosseland opacity, to be read on the RHS axis.

properties. Currently, the effects of such competing processes can only be observed in the atmospheric layers. It turns out that the specific properties of pulsating hot subdwarf stars may in this context become of particular interest. This is linked to the main factor that effectively controls the efficiency of the pulsation engine, as we outline below.

Considering a representative sdB star with parameters $T_{\text {eff }}=34,000 \mathrm{~K}, \log g=5.8$, $M=0.48 M_{\odot}$, and $\log q(H) \simeq \log \left(M_{\text {env }} / M\right)=-4.0$, typical for an object located near the center of the EC 14026 instability domain, Figure 4 shows various model variants: a second generation model incorporating iron profiles predicted from diffusive equilibrium (hereafter the "reference model") and a series of structures (hereafter called "Z-bump enhanced models") built to explore the effect of increasing the amount of iron only in the Z-bump region, while keeping it solar everywhere else in the envelope. For that purpose, the iron abundance distribution in the envelope was artificially set to a step-like profile centered at the tip of the iron opacity peak $(\log q \sim-10$ in the present case), extending down to $\log q=-9$ and up to $\log q=-11$, and connecting with exponential tails to the uniform solar value in order to fully cover the region occupied by the Z-bump. The height of the step profile is defined by a factor that is applied to the reference iron content, the latter being the solar value $\log \mathrm{Fe} / \mathrm{H}=-4.5$. Such models are obviously unphysical and should not be used for precise asteroseismic purposes, but they turn out to be particularly interesting, however, for pinning down the main contributing factor controling the efficiency of the pulsation engine. 
Nonadiabatic pulsation calculations on this series of models indicate that the same band of excited modes as in the reference model is found for Z-bump enhanced models with $\times 12$, $\times 17$, and $\times 22$ iron enrichments (green solid curves in Figure 4). Models with lower (higher) enhancement factors are found to excite a narrower (wider) band. An important fact, and this clearly appears in the upper left panel of Figure 4, is that the amounts of iron enrichment in the $\times 12, \times 17$, and $\times 22$ models correspond approximately to the total content of iron that is levitating in the Z-bump region according to diffusive equilibrium calculations included in the reference model. A closer look at the reference model indicates that the quantity of iron levitating near $\log q \sim-10$ (i.e., at the top of the opacity bump) is indeed $\sim 12.11 \times$ the solar value. In complement, we show in the right panel of Figure 4 the driving/damping structure for a representative $\ell=0, k=2$ mode in the reference model (upper panel) and in a Z-bump enhanced model with an iron enrichment factor of $\times 12.11$ (lower panel). This plot illustrates the similar shapes of the Z-bump structures in the opacity profiles for these two models. In addition, it demonstrates, through the similar shapes of the integrand of the work integral associated with this mode (the red solid curves), that the mode driving structure is also essentially unchanged. We recall that this quantity measures the energy locally gained (lost) in each stellar layer by the mode over a pulsation cycle. Positive values indicate local driving and negative values show the damping regions.

The important conclusion is that the strength of the pulsation engine at work in hot subdwarf stars is essentially controled by a single factor, the latter being largely dominated by the amount of opaque metals (iron and eventual additional contributors such as nickel) present in the Z-bump region only. The chemical composition in other stellar layers turns out to be irrelevant as far as mode excitation is concerned, although it plays an essential role for precise determinations of the pulsation periods. In other words, the nonadiabatic properties of pulsating hot subdwarfs only depend on the metal content present in the very localized subphotospheric layers where the Z-bump occurs, regardless of the physical process that ultimately leads to such local abundances.

\section{Probing the metal content in the Z-bump region}

The above conclusion suggests a new nonadiabatic asteroseismic approach to the study of hot subdwarf stars. The upper panel of Figure 5 illustrates the basics of this approach for the representative sdB star model mentioned in the previous section. On the left hand side of this plot, the period spectrum for the reference model is represented vertically, with the driven modes shown as blue filled circles. The other sequences of period spectra are from Z-bump enhanced models with, from left to right, increasing values for the iron enrichment factor. This figure allows to identify two interesting events in terms of mode stability. The first one, occuring between enrichments factors of $\times 3$ and $\times 3.1$, is the onset of mode instabilities. Below an enrichment factor of $\times 3.1$, no pulsations are expected in these stars. This sets a limit of the amount of levitating iron that must be present in the Z-bump region for both pulsating (above) and nonpulsating (below) sdB stars with parameters $T_{\text {eff }} \sim 34,000 \mathrm{~K}$ and $\log g \sim 5$.8. The second event is reached when increasing further the enrichment factor. The domain of excited periods then naturally expands, mainly to modes with higher radial orders, until it reaches a point where the band of unstable modes corresponds to the one seen in the reference model. As shown in Figure 5 (models with green filled circles), this happens in a limited range - between $\times 11$ and $\times 22.7$ - for the enrichment factor. We recall that a factor $\times 12.11$ of iron is indeed levitating at the tip of the opacity bump in the reference model.

The same argumentation can be transposed to real pulsators assuming that the sensitivity of the observations has been sufficient to determine the whole range of driven modes in these stars. Another assumption is that all modes predicted to be unstable by the nonadiabatic linear pulsation theory can indeed be excited to observable amplitudes in the real star. The validity of this assumption, or possible corrections to it, rely on a detailed nonlinear description of 

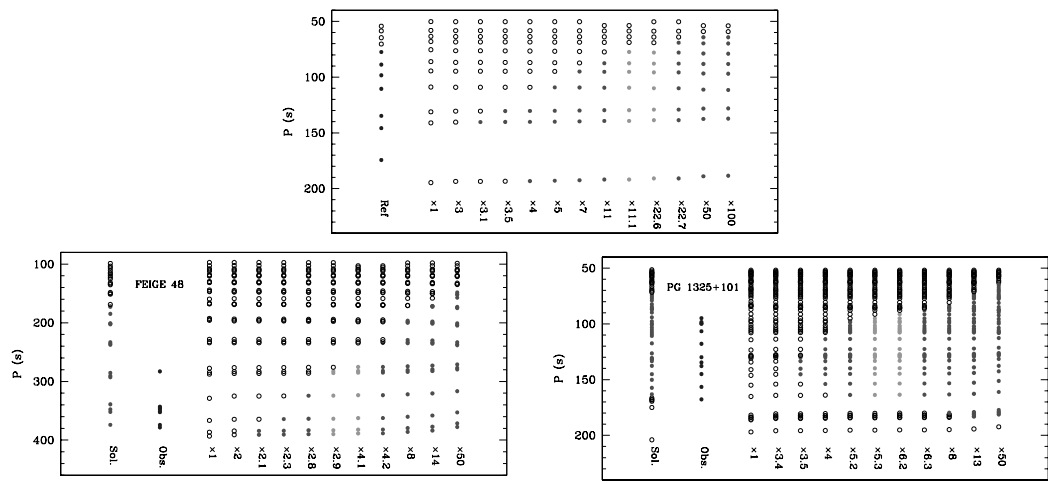

Figure 5: Upper panel: The period spectrum of the reference model is shown on the LHS of this plot. Other models are Z-bump enhanced structures with varying iron enrichment factors. Black open circles indicate damped modes and colored filled circles correspond to driven modes. Blue is used for the reference model, red for models that do not match the range of excited modes in the reference model, and green for models that match this range. Lower panels: Nonadiabatic asteroseismology of the short-period sdB pulsators Feige 48 (lower left panel) and PG 1325+101 (lower right panel). Blue filled circles now indicate the observed periods, the model on the LHS is the optimal solution derived from asteroseismology, and other models are Z-bump enhanced structures with various enrichment factors. Series with green filled circles indicate models that match the range of observed modes in the considered star.

stellar oscillations which, however, is currently not available. With these possible limitations in mind, we illustrate the method in the lower panels of Figure 5 for two well-studied short period sdB stars: PG $1325+101$ at $T_{\text {eff }} \simeq 35,050 \mathrm{~K}, \log g \simeq 5.811$ (Charpinet et al. 2006) and Feige 48 at $T_{\text {eff }} \simeq 29,580 \mathrm{~K}, \log g \simeq 5.462$ (Van Grootel et al. 2008). The observed period spectrum for each star is given as blue filled circles. It is compared with the period spectrum of their respective best model solutions proposed in the above cited publications. In both cases, the range of excited periods in that model is clearly more extended than the observed range, a typical situation with the second generation models. By producing two series of Z-bump enhanced models, each adapted to the star under consideration, we can determine the iron content needed, first, to provoke the onset of pulsation mode instabilities (this occurs at factors of $\times 3.5$ and $\times 2.1$ for PG $1325+101$ and Feige 48 , respectively, thus showing that this limit varies as a function of $\log g$ and $T_{\text {eff }}$ ) and, second, to match the observed period range. The latter occurs in the ranges $\times 5.2-6.3$ for PG $1325+101$ and $\times 2.3-2.9$ for Feige 48. Hence, through the new nonadiabatic asteroseismic approach, we obtain independent estimates for the amount of iron present at the location of the Zbump, regardless of the physical process involved to create this particular abundance. For PG $1325+101$ we estimate an iron enrichment of $\times 5.75 \pm 0.55$ over the solar value, i.e., $\log \mathrm{Fe} / \mathrm{H}=-3.74 \pm 0.04$. For Feige 48, we find an enrichment of $\times 2.60 \pm 0.30$ over the solar value, i.e., $\log \mathrm{Fe} / \mathrm{H}=-4.09 \pm 0.05$.

The values given just above only illustrate the potential of the method. At this stage, they should not be taken at face value, as they are likely subject to systematic effects that need to be investigated in detail. These effects are mainly related to the facts that differences between OPAL and OP opacities may be sufficiently large to possibly affect significantly the derived values, and the contribution of other species, in particular nickel, to the opacity bump may be nonnegligible. The uncertainty associated with the opacities should disappear after the issue concerning which, of the OPAL or OP data set, is the most realistic one is settled. At this stage, we simply note that using OP opacities instead of the OPAL data, as we did 
here, may lead to a decrease of the derived values for the iron abundance because the driving mechanism appears more efficient for a given amount of iron in the Z-bump region when OP data are used (see Jeffery \& Saio 2007). It is impossible at this stage to say if this change will be significant or not. We also stress that if nickel has an important contribution to the Z-bump opacity and to the driving mechanism, the basic principle of the proposed method still applies. The measured quantity, however, would then correspond to the mixture of $\mathrm{Fe}+\mathrm{Ni}$ that would be present in the driving region (instead of only iron as given in our examples above). This would mean, for given opacities and provided that the $\mathrm{Ni} / \mathrm{Fe}$ ratio can be estimated from other considerations, that both the amount of nickel and the quantity of iron present in that region could potentially be estimated. Addressing the above-mentioned issues will require further exploration of these effects using improved sets of models.

\section{Conclusion and prospects}

We reviewed and illustrated some of the basic properties of the driving mechanism responsible for pulsations in hot subdwarf stars. In particular, we outlined that the pulsation engine at work in these stars is predominantly controlled by the amount of metals (mostly iron and possibly nickel) present in the subphotospheric layers where the Z-bump occurs, irrespective of the precise mechanism responsible for building up such an abundance. The chemical composition in other parts of the star is essentially irrelevant to the process. This remarkable property is of high interest as it opens up a new independent way for probing the interior of hot subdwarf stars, through a nonadiabatic asteroseismic approach. This nonadiabatic method should constitute a very useful complementary approach to the study of abundance anomalies in hot subdwarf stars. More generally, it may help to shed new light on the effects of various mixing processes that may be at work in stars in general. In this context, pulsating hot subdwarfs may then constitute precious laboratories to progress further on these problems.

Acknowledgments. S. Charpinet thanks the SOC for its invitation to give this review talk and is grateful for the financial support offered by the HELAS Consortium.

\section{References}

Blanchette, J.-P., Chayer, P., Wesemael, F., et al. 2008, ApJ, 678, 1329

Charpinet, S., Fontaine, G., Brassard, P., \& Dorman, B. 1996, ApJ, 471, L103

Charpinet, S., Fontaine, G., Brassard, P., et al. 1997, ApJ, 483, L123

Charpinet, S., Silvotti, R., Bonanno, A., et al. 2006, A\&A, 459, 565

Charpinet, S., Fontaine, G., Brassard, P., et al. 2007, CoAst, 150, 241

Dupret, M.-A.,2008, CoAst, 157, 16

Fontaine, G., Brassard, P., Charpinet, S., et al. 2003, ApJ, 597, 518

Fontaine, G., Brassard, P., Charpinet, S., \& Chayer, P. 2006a, MmSAI, 77, 49

Fontaine, G., Green, E. M., Chayer, P., et al. 2006b, BaltA, 15, 211

Fontaine, G., Brassard, P., Charpinet, S., et al. 2008a, ASPC, 392, 231

Fontaine, G., Brassard, P., Green, E., et al. 2008b, A\&A, 486, L39

Fontaine, G. \& Brassard, P. 2008, CoAst, 157, 177

Green, E. M., Fontaine, G., Reed, M. D., et al. 2003, ApJ, 583, L31

Jeffery, C. S., \& Saio, H. 2006a, MNRAS, 371, 659

Jeffery, C. S., \& Saio, H. 2006b, MNRAS, 372, L48

Jeffery, C. S., \& Saio, H. 2007, MNRAS, 378, 379

Kilkenny, D., Koen, C., O'Donoghue, D., \& Stobie, R. S. 1997, MNRAS, 285, 640

Van Grootel, V., Charpinet, S., Fontaine, G., \& Brassard, P. 2008, A\&A, 483, 875

Woudt, P. A., Kilkenny, D., Zietsman, E., et al. 2006, MNRAS, 371, 1497 


\section{DISCUSSION}

Dziembowski: Is there any role of the "deep opacity bump" in driving pulsation of the O subdwarf ?

Charpinet: There is no evidence of significant driving from the "deep opacity bump", at least for the modes corresponding to the periods observed in the pulsating sdO star

Noels: In WR stars, the main driving comes from the deep opacity bump. If iron is increased near that bump, it maybe could add a contribution to the driving. Of course, there can be a problem with the frequency range.

Charpinet: It doesn't seem to be the case in the model for the unique pulsating sdO star that we know, but it may possibly contribute to the excitation of modes in a different frequency range. This potential driving needs further investigation, though, and there is currently no observational evidence of sdO stars pulsating in another range of frequencies.

Zahn: Do your models, with just radiative levitation and gravitational settling, predict the observed surface abundances, or do you have to invoke some turbulent diffusion?

Charpinet: Reproducing precisely the observed surface abundances in sdB stars is difficult. Just for helium, radiative levitation and gravitational settling alone do not account for the observed surface abundance. Other competing processes should clearly play a role. For helium, weak stellar winds are usually invoked, but other mixing or diffusive processes may contribute as well. Of course, what is true for helium is also applicable to other chemical species. There is still work to do to reach full consistency at that level and asteroseismology should be a precious guide here.

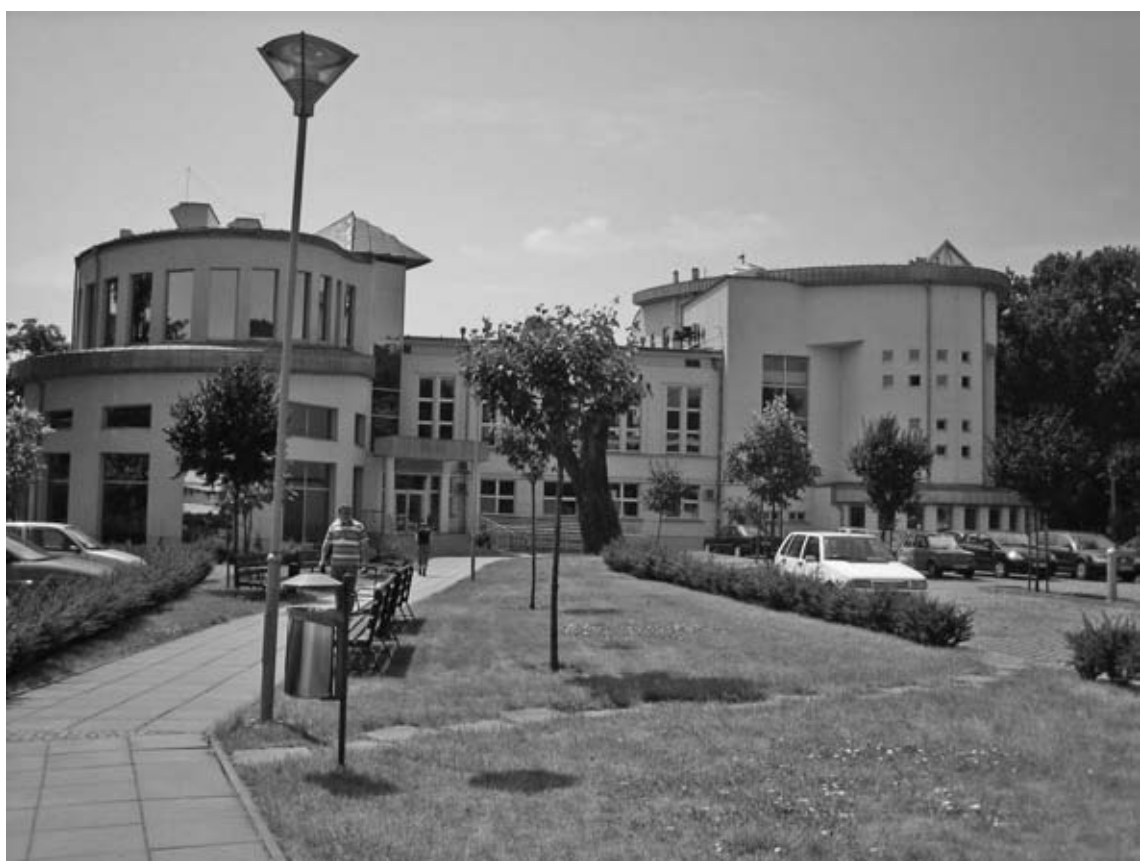

The conference venue 
Comm. in Asteroseismology

Vol. 157, 2008, Wroctaw HELAS Workshop 2008

M. Breger, W. Dziembowski, \& M. Thompson, eds.

\title{
The degenerate pulsators
}

\author{
G. Fontaine ${ }^{1}$ and P. Brassard ${ }^{1}$ \\ ${ }^{1}$ Département de Physique, Université de Montréal, C.P. 6128, \\ Succ. Centre-Ville, Montréal, Québec, Canada H3C 3J7
}

\begin{abstract}
We briefly review some of the basic properties of the three distinct families of isolated pulsating white dwarfs. These are the GW Vir stars ( $\mathrm{He} / \mathrm{C} / \mathrm{O}$-atmosphere stars with $T_{\text {eff }} \sim 120,000 \mathrm{~K}$ ), the V777 Her stars (He-atmosphere, $T_{\text {eff }} \sim 25,000 \mathrm{~K}$ ), and the ZZ Ceti stars ( $\mathrm{H}$-atmosphere, $T_{\text {eff }} \sim 12,000 \mathrm{~K}$ ), all showing multiperiodic luminosity variations caused by low-order and low-degree g-mode instabilities. In the light of the recent and unexpected discovery of a new type of white dwarfs, those with atmospheres dominated by carbon and found only in a narrow range of effective temperature centered around $20,000 \mathrm{~K}$, we also review the asteroseismological potential of these objects. This sets the table for a discussion of the recent exciting discovery of the first pulsating C-atmosphere white dwarf, SDSS J1426+5752.
\end{abstract}

\section{The white dwarf stars as compact pulsators}

The white dwarfs are the end products of the evolution of more than $97 \%$ of all stars. Stars with initial masses on the main sequence of $\sim 8 M_{\odot}$ and less end up as white dwarfs in a narrow range of mass, from about 0.4 to $1.2 M_{\odot}$, with a sharply peaked distribution centered around $\sim 0.6 M_{\odot}$. This implies important mass loss in previous evolutionary phases (red giant phases). White dwarfs have run out of thermonuclear fuel and most of them are constituted of a C/O core - the products of $\mathrm{H}$ and He burning - containing some $99 \%$ of the total mass. They are cooling bodies in hydrostatic equilibrium; gravity is balanced by degenerate electron pressure. This implies an evolution at almost constant radius. White dwarfs obey the famous mass-radius relation put forward by Chandrasekhar in the 1930's. They shine through the slow leakage of thermal energy of the ionic plasma, and the latter, making up a gas in the early stages of white dwarf evolution, is best described in terms of a liquid and, ultimately, a solid as cooling proceeds (see, e.g., Fontaine, Brassard, \& Bergeron 2001 for a complete review of white dwarf properties).

About 2200 field white dwarfs are listed in the McCook and Sion Catalog (McCook \& Sion 1999), but more than 10,000 are now known spectroscopically through the SDSS project. The average visual magnitude of the stars in the bright McCook and Sion sample is $\langle\mathrm{V}\rangle \simeq 15.5$. White dwarfs have radii in the range from $\sim 0.025$ to $\sim 0.006 R / R_{\odot}$, and, combined with the above mass interval, this leads to a range of surface gravity $g$ from $\sim 10^{7}$ to $\sim 10^{9} \mathrm{~cm} \mathrm{~s}^{-2}$. A typical average density in a white dwarf is thus $\sim 10^{6} \mathrm{~g} \mathrm{~cm}{ }^{-3}$, compared with a value of $\sim 1 \mathrm{~g} \mathrm{~cm}^{-3}$ in a normal star. Finally, white dwarfs are found in the full range of effective temperature in the HR diagram, from $3000 \mathrm{~K}$ to $200,000 \mathrm{~K}$, and their luminosities span more than seven orders of magnitude, with $\log \left(L / L_{\odot}\right)$ ranging from -4.7 to 2.5 . 


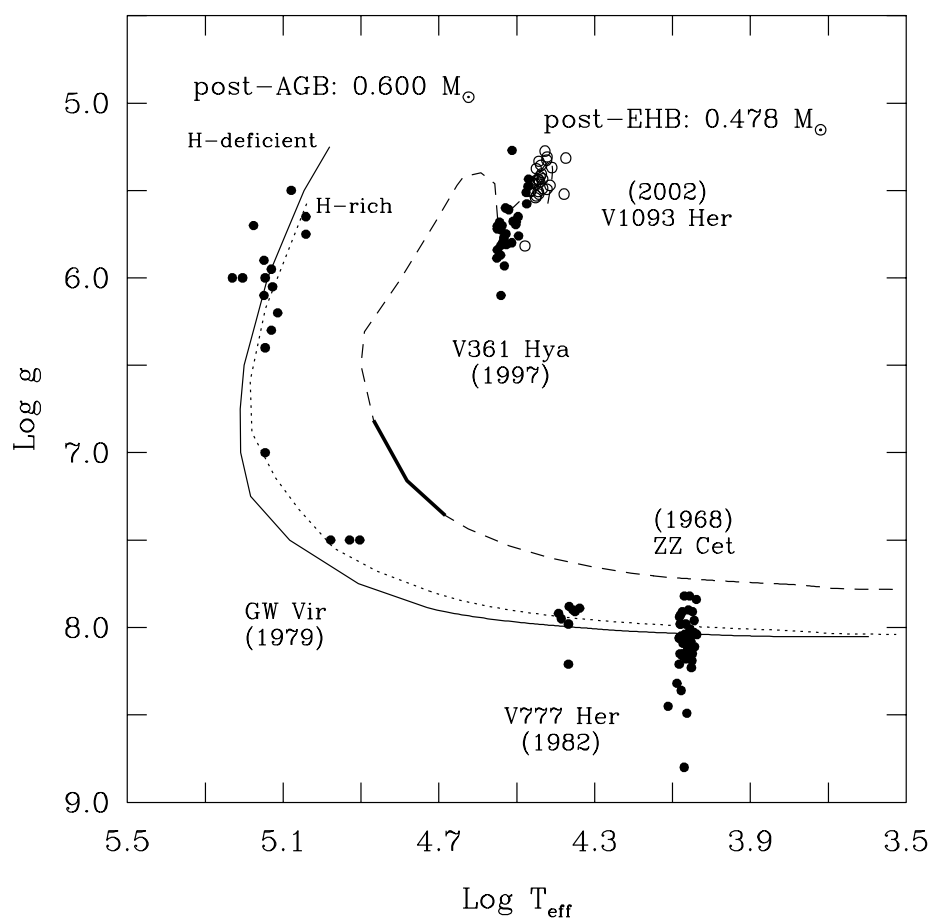

Figure 1: Region of the $\log g-\log T_{\text {eff }}$ plane where the compact pulsators are found. Each of the five distinct families is identified by its official IAU name. The year that the discovery of the prototype of each class is reported, is also indicated. Typical evolutionary tracks are plotted showing 1) the track followed by a $0.6 M_{\odot}$ post-AGB, $\mathrm{H}$-rich star which becomes a $\mathrm{H}$-atmosphere white dwarf (dotted curve), 2) the path followed by a $0.6 M_{\odot}$ post-AGB, $\mathrm{H}$-deficient star which becomes a He-atmosphere white dwarf (solid curve), and 3 ) the path followed by a $0.478 M_{\odot}$ post-EHB model which leads to the formation of a low-mass $\mathrm{H}$-atmosphere white dwarf (dashed curve).

Most white dwarfs descend from post-AGB evolution, while a small minority, less than about $2 \%$, descend from post-EHB evolution (see, e.g., Figure 1). About $80 \%$ of post-AGB stars manage to retain some $\mathrm{H}$ in their outermost layers, and those give rise, through efficient gravitational settling ( $\log g \sim 8)$, to essentially pure $\mathrm{H}$-atmosphere or DA white dwarfs. The dotted curve in Figure 1 illustrates the evolutionary path followed by a $0.6 M_{\odot}$, post-AGB, $\mathrm{H}$-atmosphere white dwarf in the spectroscopic HR diagram. The other $20 \%$ of post-AGB objects undergo a late helium flash during which the residual hydrogen is burned away, thus giving rise to the other family of white dwarfs, those with He-atmospheres. The solid curve is representative of the evolutionary path of such a He-atmosphere or non-DA white dwarf.

During its evolution, a He-atmosphere white dwarf may become a pulsator twice. First, in the very hot early phases, it may pulsate as a GW Vir star, and then later on, it may pulsate as a DB or V777 Her star in a narrow instability strip centered around 25,000 K (see Figure 1). For their part, $\mathrm{H}$-atmosphere white dwarfs all pulsate when they go through another narrow instability strip, this time centered around $12,000 \mathrm{~K}$, the ZZ Ceti instability strip. In all three cases, low-degree and low- to mid-order g-modes are involved. Figure 1 


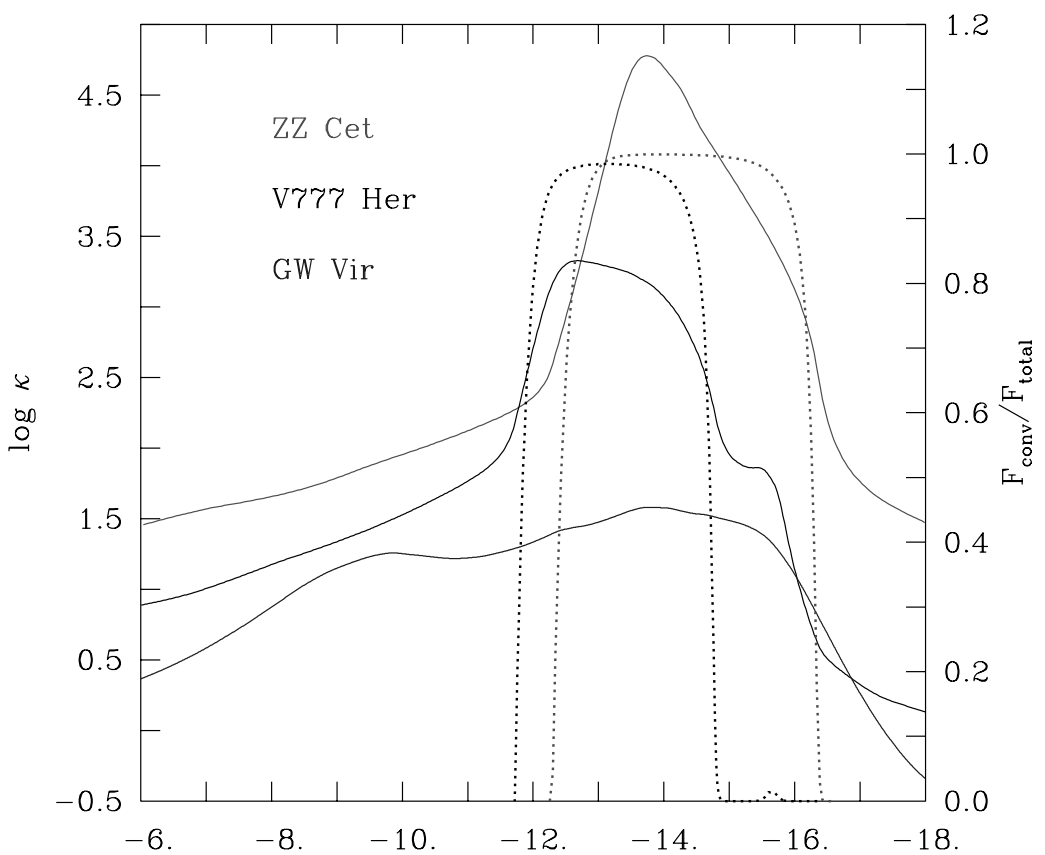

$\log \mathrm{q}=\log \left(1-\mathrm{M}(\mathrm{r}) / \mathrm{M}_{*}\right)$

Figure 2: Opacity profiles in the envelopes of representative models of GW Vir (lower solid curve), V777 Her (middle solid curve), and ZZ Ceti (upper solid curve) stars. The abcissa is expressed as the logarithm of the fractional mass above the point of interest. The center of the model would be located at a value $\log q=0$ on that scale, but we have chosen to emphasize here only the outermost layers where the action is concentrated in terms of driving/damping in white dwarfs. The ratio of the convective to total flux is alo plotted for the V777 Her (dotted curve on the left) and the ZZ Ceti model (dotted curve on the right). There is no convection in the very hot GW Vir star model.

also depicts the locations of two other families of compact pulsators, the short- (V361 Hya) and long-period (V1093 Her) pulsating hot subdwarf B stars, which are not yet white dwarfs and, therefore, will no longer be considered in this paper. Several interesting communications about the latter pulsators have been presented at this Conference.

The phenomenon ultimately responsible for driving pulsation modes in white dwarfs is the recombination of the main envelope constituents. GW Vir stars have envelopes made up of $\mathrm{He}, \mathrm{C}$, and $\mathrm{O}$ in roughly comparable proportions (Werner \& Herwig 2006), and it is the partial ionization of the $\mathrm{K}$-shell electrons in $\mathrm{C}$ and $\mathrm{O}$ that is at work in these pulsators. In the case of V777 Her (ZZ Ceti) stars, it is the recombination of Helll and Hell (HII) that ultimately triggers pulsational instabilities. And indeed, partial ionization leads to the formation of an opacity bump in the envelope which acts as a bottleneck for photons trying to escape.

Figure 2 illustrates the opacity profile in the envelope of a representative model of each of the three types of pulsating white dwarfs. The lower solid curve shows the opacity profile for a typical model of a GW Vir pulsator. In that case, the opacity bump is relatively small in magnitude, there is no associated convection, and the related driving is a classical $\kappa$-mechanism. 


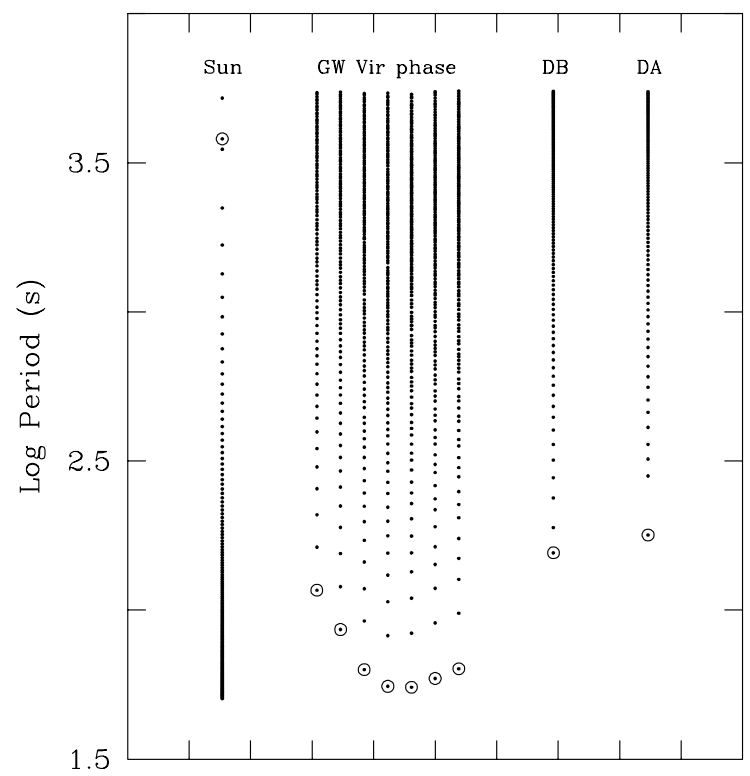

Figure 3: Comparison of the period spectra for modes with $\ell=1$ in the period interval $50-5500 \mathrm{~s}$ for 1) a solar model (left column), 2) seven evolving GW Vir models (the seven columns bunched together), 3) a V777 Her model, and 4) a ZZ Ceti model (right column). The circle in each column separates the $g$-branch above from the $p$-branch below and identifies the first overtone $(k=1)$ of the $g$-branch. All of the white dwarf models considered have the same total mass of $0.6 M_{\odot}$.

Note that the "active" bump is the lower one centered around a fractional mass depth of $q \simeq 10^{-10}$, while the upper one is the usual "Z-bump" located too far up in the envelope to contribute significantly to driving or damping. In V777 Her stars (middle solid curve) and, a fortiori, in ZZ Ceti stars (upper solid curve), the opacity bump is much larger, and this leads to the formation of a concomitant convection zone. In this connection, the dotted curves in Figure 2 show the profiles of the ratio of the convective to total flux for these two types of pulsating white dwarfs. In those cases, the excitation mechanism is no longer the classical $\kappa$-mechanism as modulations of the convective flux now play a major role. The main driving region is then located at the base of the convection zone, and one speaks of convective driving.

To understand pulsations in white dwarfs, it is first necessary to realize that their mechanical structure is fundamentally different from that of normal stars. On the first account, the compact nature of a white dwarf implies that the period of a pulsation mode ( $p$ or $g$ ) defined by its radial order $k$ and its degree index $\ell$ is much smaller than in normal stars. This is shown in Figure 3 where the dipole mode period spectrum of a solar model in the range of periods from $50 \mathrm{~s}$ to $5500 \mathrm{~s}$ - the interval in which periods of pulsating white dwarfs are found - is compared to those of representative models for each of the three types of white dwarf pulsators. Except for the two longest periods shown in the figure for the solar model, all of the other modes depicted for that model are $p$-modes. This is just the opposite for the degenerate star models as the modes falling in the period window of interest are all $g$-modes. 
It should be noted that the fact that the observed periods (more typically $100 \mathrm{~s}$ to $1000 \mathrm{~s}$ in high-gravity GW Vir stars, in V777 Her stars, and ZZ Ceti stars) are relatively short should be considered a good thing, although photon starvation is always a problem for white dwarfs. This is because several pulsation cycles can be covered in a single night of observations, allowing for a much better assesment of the multiperiodic nature of the light curves.

On the second account, it should be known that $g$-modes propagate readily in the outermost layers in degenerate stars and show maximum amplitudes there. We noted above that a typical white dwarf is constituted of a C/O core making up more than $99 \%$ of the total mass. That core is usually surrounded by a thin He mantle that contains less than $1 \%$ of the total mass. In DA white dwarfs (about $80 \%$ of degenerate stars), there is also an outermost $\mathrm{H}$ envelope that contains less than $0.01 \%$ of the total mass. Although extremely thin, these onion-like layers are of fundamental importance in the asteroseismology of white dwarfs because the $g$-modes have substantial amplitudes only in those outer regions. Because of this, the layered chemical structure of a white dwarf leads, through mode trapping/confinement, to a highly nonuniform period distribution for $g$-modes belonging to the same degree index $\ell$ but with different values of $k$. This is again a good thing because it suggests that chemical layering could potentially be measured accurately through asteroseismological means.

Finally, it should be realized that the degenerate interior of a white dwarf is refractory to asteroseismological probing as modes tend to be formed outside the most degenerate central regions. Those regions indeed contribute very little to the weight function integral. This is a problem for probing the $\mathrm{C} / \mathrm{O}$ core directly through period measurements. With cooling, overall degeneracy increases and the region of mode formation migrates outward. This implies that the pulsation modes of a degenerate star progressively loose their ability to probe the deep interior (as it is still possible in a GW Vir star), and become more sensitive to the details of the outermost layers (in the ZZ Ceti regime, for example). The type of asteroseismology that is possible with a GW Vir star is not exactly the same as that possible with a ZZ Ceti pulsator. This seems to have been not appreciated by many in the past. More on this and several other aspects of pulsating white dwarfs can be found in a comprehensive review paper that we wrote recently (Fontaine \& Brassard 2008).

\section{A new type of pulsating white dwarf}

In the context of this conference, it is most appropriate to mention an exciting development that came up recently on the scene of white dwarf asteroseismology. This resulted from the unexpected discovery of a new type of white dwarfs, those with carbon-dominated atmospheres, also known as Hot DQ stars (Dufour et al. 2007). These are very rare, and Dufour et al. (2007) reported the discovery of 9 of those, out of a total of about 10,000 white dwarfs identified spectroscopically. The Hot DQ stars were uncovered within the framework of the SDSS project. Among other characteristics, they all bunch together in a narrow range of effective temperature centered around 20,000 K (Dufour et al. 2008a).

It has been known for quite some time (see, e.g., Fontaine \& Van Horn 1976) that models of carbon-atmosphere white dwarfs in this temperature range possess superficial convection zones that bear strong similarities with those found in $\mathrm{H}$ - and He-atmosphere stars. Given that the newly-found C-atmosphere white dwarfs are sandwiched between the V777 Her and ZZ Ceti instability strips, and given that convection plays a key role in the excitation of pulsation modes in these pulsators, the prospects of finding unstable models of Hot DQ stars looked promising at the outset.

We therefore carried out an exploratory stability survey of models of carbon-atmosphere white dwarfs using a full nonadiabatic approach (Fontaine et al. 2008). Our theoretical survey has revealed that $g$-modes can indeed be driven in models of Hot DQ stars. However, we found that only those stars with a sufficiently large amount of $\mathrm{He}(X(\mathrm{He})>0.25)$ in their 


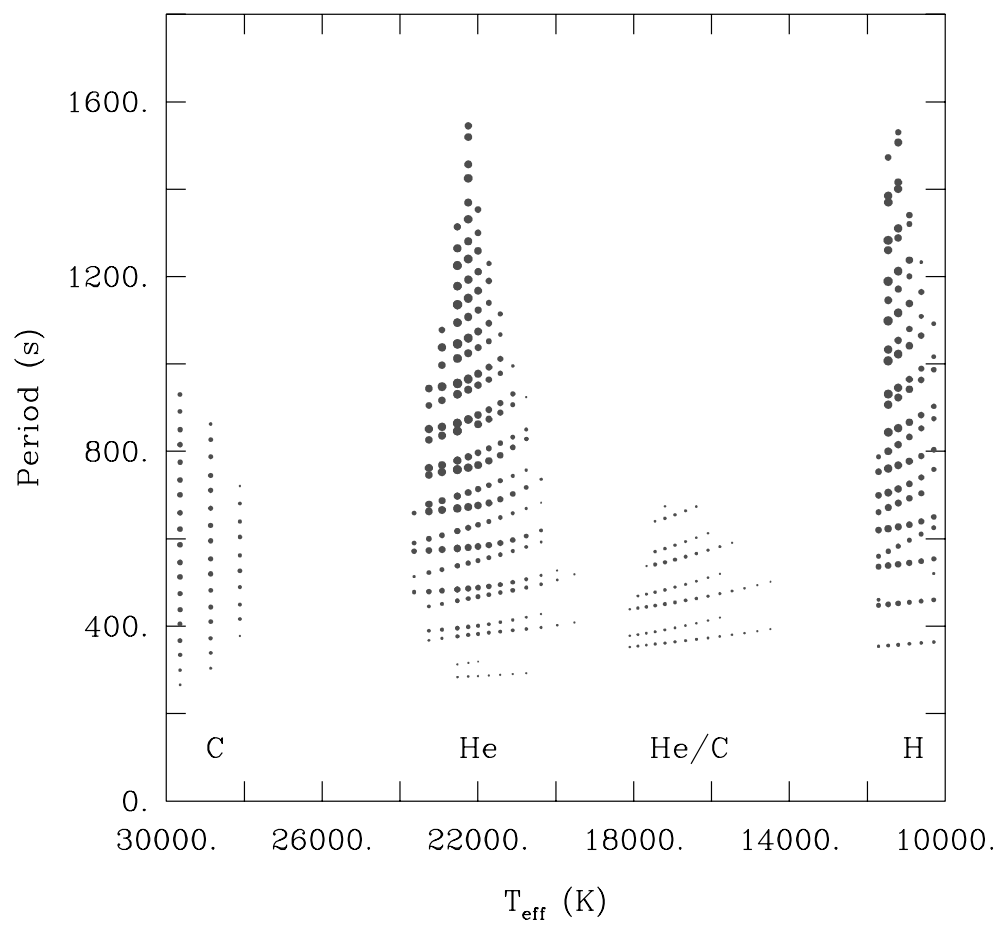

Figure 4: Predicted spectra of excited $g$-modes computed from four distinct evolutionary sequences, each characterized by a total mass of $0.6 \mathrm{M}_{\odot}$, but with a different envelope composition: pure $\mathrm{C}$, pure $\mathrm{He}$, $X(\mathrm{He})=X(\mathrm{C})=0.5$, and pure $\mathrm{H}$, from left to right. The size of each plotted point gives a logarithmic measure of the imaginary part of the complex eigenfrequency for a given excited mode; the bigger the dot, the more unstable the mode.

C-rich envelope mixture could pulsate in the range of effective temperature where the real C-atmosphere white dwarfs are found.

In this connection, Figure 4 displays some revealing results. It depicts the locations of theoretical instability strips for evolving $0.6 M_{\odot}$ white dwarf models with different envelope compositions. Along with the usual V777 Her (pure $\mathrm{He}$ ) and ZZ Ceti (pure H) instability strips, one can recognize the red edge of the pulsating pure $C$ envelope white dwarf models. In fact, the pure $\mathrm{C}$ instability strip extends all the way up to the GW Vir regime as described at length in Quirion et al. (2007). Given that the true red edge is hotter than the $\sim 28,000$ $\mathrm{K}$ value found in our survey because of convection/pulsation interactions (see Fontaine \& Brasssard 2008), it follows that pure C-atmosphere white dwarfs cannot pulsate in the range of effective temperature where the Hot $D Q$ stars congregate.

On the other hand, models with a mixed $\mathrm{He}$ and $\mathrm{C}$ envelope composition can also pulsate, but in different temperature intervals. For instance, Figure 4 illustrates a new instability strip between the V777 Her and the ZZ Ceti domains associated with white dwarf models with a mixed envelope composition specified by $X(\mathrm{He})=X(\mathrm{C})=0.5$. Naively, one could have expected to find such a strip in between the pure $C$ and pure He strips, but structural differences in the mixed envelope composition models explain why this is not the case. In brief, 


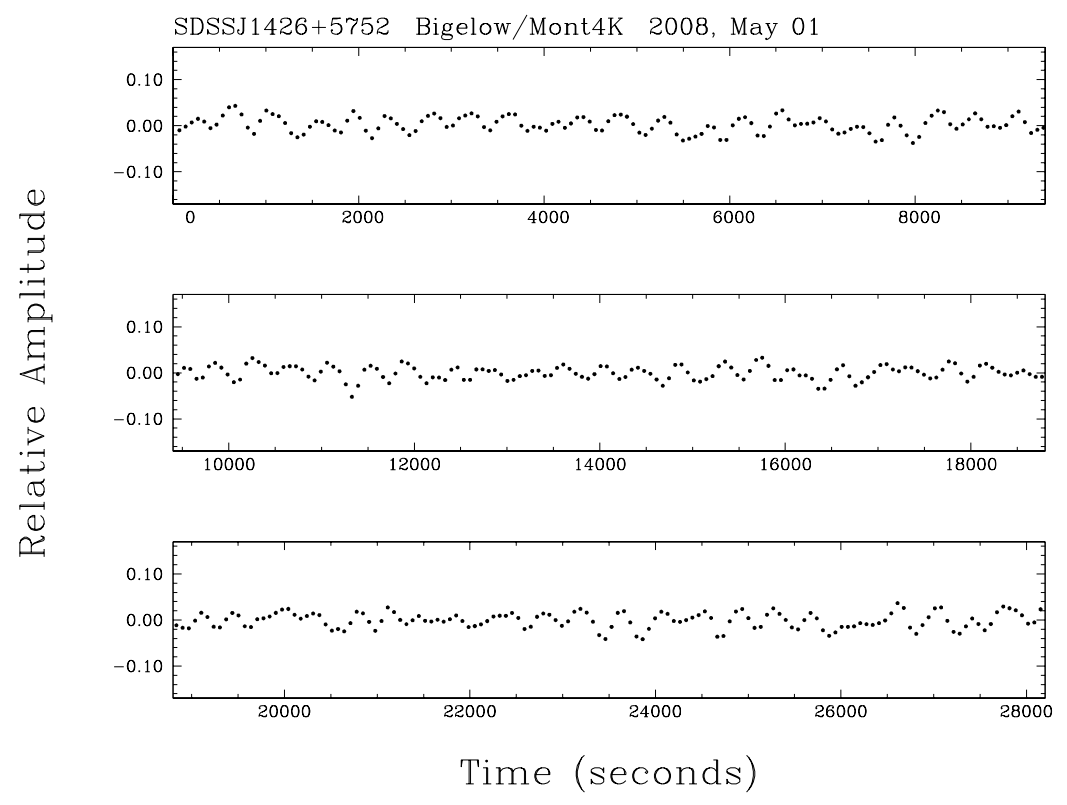

Figure 5: Sample broadband light curve of SDSS J1426+5752 obtained with the Mont4K/Bigelow combination. Each plotted point corresponds to an effective sampling time of $67 \mathrm{~s}$.

the survey of Fontaine et al. (2008) revealed that some Hot DQ white dwarfs can indeed undergo low-order, low-degree g-mode pulsational instabilities, provided that the surface gravity is larger than average and a substantial amount of $\mathrm{He}$ is present in the C-rich envelope mixture.

In parallel with our theoretical investigations, but totally independently, Montgomery et al. (2008) carried out an observational search for luminosity variations in six Hot DQ stars accessible to observations in the winter of 2008. They were able to report the very exciting discovery that SDSS J1426+5752, one of the Hot DQ's found by Dufour et al. (2007), pulsates in at least one mode with a period of $417 \mathrm{~s}$, thus establishing the existence of a fourth type of pulsating white dwarf. Full credit should be given to Montgomery et al. (2008) for this important breakthrough. In addition, they reported that no luminosity variations were found, to the limit of detection, in the five other stars in their sample.

Following this discovery, Green, Fontaine, \& Dufour undertook follow-up wide band photometric observations of SDSS J1426+5752 at the Steward Observatory $1.6 \mathrm{~m}$ telescope on Mount Bigelow with the help of the new Montréal $4 \mathrm{~K} \times 4 \mathrm{~K}$ CCD camera (Mont4K), a joint venture between the University of Arizona and the Université de Montréal. Some $106 \mathrm{~h}$ of observations were obtained on this rather faint star $(g=19.2)$. Figure 5 illustrates one of the nightly light curves obtained by Green et al., leaving no doubt as to the variability of SDSS $\mathrm{J} 1426+5752$. The subsequent Fourier analysis of the full data set confirmed the presence of a dominant pulsation with a period of $417 \mathrm{~s}$ and of its first harmonic as first reported by Montgomery et al. (2008). Furthermore, it also revealed the presence of an additional 
pulsation with a period of $319 \mathrm{~s}$ at the 4.9 sigma level. Hence, with at least two independent periodicities uncovered, SDSS J1426+5752 can be considered as a multiperiodic pulsator like the other types of pulsating white dwarfs.

In view of the rather poor SDSS spectrum available for SDSS J1426+5752, follow-up spectroscopic observations were pursued by Dufour et al. (2008b) using both the MMT and one of the Keck telescopes. The objective was, firstly, to obtain a sufficiently good spectrum for detailed atmospheric modeling and, secondly, to search for the presence of He required to account for the observed pulsational instabilities according to the nonadiabatic calculations of Fontaine et al. (2008). The spectral analysis of the improved spectra readily revealed the presence of a substantial amount of helium in the atmosphere of SDSS J1426+5752, an abundance comparable to that of carbon by mass fraction. This is in line with the expectations of nonadiabatic pulsation theory which require an important He "pollution" in the atmosphere/envelope of SDSS J1426+5752 for it to pulsate at its current effective temperature. At the same time, the spectroscopic study of Dufour et al. (2008a) has revealed that there is not enough He pollution in the atmospheres of the other five Hot DQ stars investigated for variability by Montgomery et al. (2008) for them to pulsate. And, indeed, none were found to pulsate.

To add to this small success, an unexpected surprise came out of the follow-up spectroscopic observations of Dufour et al. (2008b). It was found that the strong carbon lines seen in the spectrum of SDSS J1426+5752 feature Zeeman splitting, a structure that could not be seen in the original noisy SDSS spectrum. The observed splitting between the $\pi$ and $\sigma$ components implies a large scale magnetic field of about 1.2 MG. Hence, SDSS J1426+5752 is both a pulsating and a magnetic white dwarf. As there is no sign of binarity in either the light curve or the optical spectrum (although the phase coverage has been quite limited), SDSS $\mathrm{J} 1426+5752$ is most likely the first example of an isolated pulsating white dwarf with a large detectable magnetic field. As such, it is the white dwarf equivalent of an roAp star.

It remains to be seen if other stars similar to SDSS J1426+5752 will be found or if it will remain an isolated "freak". If we adopt a conservative point of view, it takes at least two members to define a "class", so we should perhaps refrain from referring to it as the prototype of a class for the time being. Nevertheless, SDSS J1426+5752 is certainly different from the other kinds of pulsating white dwarfs discussed in this paper. It is our hope that further data releases from the SDSS project might reveal siblings of this unique star.

Acknowledgments. We wish to thank most sincerely the members of the Scientific Organizing Committee for the invitation to present this review during the Wroclaw HELAS Workshop. G.F. also acknowledges the contribution of the Canada Research Chair Program.

\section{References}

Dufour, P., Fontaine, G., Liebert, J., et al. 2008a, ApJ, 683, 978

Dufour, P., Fontaine, G., Liebert, J., et al. 2008b, ApJ, 683, 167

Dufour, P., Liebert, J., Fontaine, G., \& Behara, N. 2007, Nature, 450, 522

Fontaine, G., \& Brassard, P. 2008, PASP, 120, 1043

Fontaine, G., Brassard, P., \& Bergeron, P. 2001, PASP, 113, 409

Fontaine, G., Brassard, P., \& Dufour, P. 2008, A\&A, 483, L1

Fontaine, G., \& Van Horn, H.M. 1976, ApJS, 31, 467

McCook, G.P., \& Sion, E.M. 1999, ApJS, 121, 1

Montgomery, M.H., Williams, K.A., Winget, D.E., et al. 2008, ApJ, 678, L51

Quirion, P.-O., Fontaine, G., \& Brassard, P. 2007, ApJS, 171, 219

Werner, K., \& Herwig, F. 2006, PASP, 118, 183 


\title{
Long-term photometric monitoring of the hybrid subdwarf B pulsator HS $0702+6043$
}

\author{
R. Lutz ${ }^{1,2}$, S. Schuh ${ }^{1}$, R. Silvotti ${ }^{3}$, R. Kruspe ${ }^{1}$, and S. Dreizler ${ }^{1}$ \\ ${ }^{1}$ Institut für Astrophysik, Friedrich-Hund-Platz 1, 37077 Göttingen, Germany \\ 2 Max-Planck-Institut für Sonnensystemforschung, Max-Planck-Straße 2, \\ 37191 Katlenburg-Lindau, Germany \\ 3 INAF - Osservatorio Astronomico di Capodimonte, via Moiariello 16, \\ 80131 Napoli, Italy
}

\begin{abstract}
Pulsating subdwarf B stars oscillate in short-period $p$-modes or long-period $g$-modes. HS $0702+6043$ is one of the three objects currently known to show characteristics of both types and hence is classified as hybrid pulsator. We briefly present our analysis of the $g$-mode domain of this star, but focus on the first results from long-term photometric monitoring in particular of the $p$-mode oscillations. We present a high-resolution frequency spectrum, and report on our efforts to construct a multi-season $\mathrm{O}-\mathrm{C}$ diagram. Additionally to the standard (although nontrivial) exercise in asteroseismology to probe the instantaneous inner structure of a star, measured changes in the pulsation frequencies as derived from an $\mathrm{O}-\mathrm{C}$ diagram can be compared to theoretical evolutionary timescales. Within the EXOTIME program, we also use this same data to search for planetary companions around extreme horizontal branch objects ("asteroseismic planets").
\end{abstract}

Individual Objects: HS 0702+6043, HS 2201+2610

\section{Introduction}

Subdwarf B stars (sdB stars) populate the extreme horizontal branch (EHB) at effective temperatures between 20000 and $40000 \mathrm{~K}$ and surface gravities $\log \left(\mathrm{g} / \mathrm{cm} \mathrm{s}^{-2}\right)$ between 5.0 and 6.2. They are believed to be core helium-burning objects of half a solar mass with remaining hydrogen envelopes too thin to sustain $\mathrm{H}$-shell burning. The reason for losing almost all of their original hydrogen envelope in earlier stages of their evolution is still unknown. The high fraction of binaries among the sdB stars suggests that close binary evolution may play an important role in their formation. The discovery of a planetary companion to HS $2201+2610$ (Silvotti et al. 2007), the only case where the necessary kind of measurements for such a discovery are available so far for an sdB star, now revives the idea that planets in wide orbits may also play a role in the formation of these stars.

A fraction of the sdB stars shows pulsations. Variable subdwarf B stars (sdBV stars) can be divided into the classes of rapid p-mode pulsators $\left(\mathrm{sdBV}_{\mathrm{r}}\right)$ and slow $g$-mode pulsators $\left(s \mathrm{dBV}_{\mathrm{s}}\right.$ ), with three objects known so far to belong to both classes simultaneously (hybrid pulsators, $\mathrm{sdBV}_{\mathrm{rs}}$ ). These are of particular interest since the two mode types probe different regions within the star. Both types of pulsations are driven by a $\kappa$-mechanism 
Table 1: Photometric data of HS0702+6043. CA: Calar Alto, T: Tübingen, SB: Steward Bok, G: Göttingen, L: Loiano, MB: Mt.Bigelow.

\begin{tabular}{lrr|lrr}
\hline \hline Date & Site & Length & Date & Site & Length \\
\hline Dec 1999 & CA $1.2 \mathrm{~m}$ & $8.4 \mathrm{~h}$ & Feb 2008 & T $0.8 \mathrm{~m}$ & $20.1 \mathrm{~h}$ \\
Feb 2004 & T $0.8 \mathrm{~m}$ & $7.3 \mathrm{~h}$ & Feb 2008 & G $0.5 \mathrm{~m}$ & $32.2 \mathrm{~h}$ \\
Feb 2004 & SB 2.2m & $12.0 \mathrm{~h}$ & Mar 2008 & G $0.5 \mathrm{~m}$ & $0.6 \mathrm{~h}$ \\
Jan 2005 & CA $2.2 \mathrm{~m}$ & $56.0 \mathrm{~h}$ & Mar 2008 & L $1.5 \mathrm{~m}$ & $8.0 \mathrm{~h}$ \\
Dec 2007 & T $0.8 \mathrm{~m}$ & $31.8 \mathrm{~h}$ & Nov07 - Mar08 & MB $1.55 \mathrm{~m}$ & $424.0 \mathrm{~h}$ \\
Dec 2007 & G $0.5 \mathrm{~m}$ & $12.0 \mathrm{~h}$ & May 2008 & G 0.5m & $4.1 \mathrm{~h}$ \\
\hline
\end{tabular}

where the required opacity bump is due to iron and nickel accumulated by diffusion, resulting in phase-stable pulsational behaviour (Charpinet et al. 1997; Fontaine et al. 2003; Jeffery \& Saio 2006).

The $p$-mode pulsators show low amplitudes (few ten mmag) and short periods (few minutes) at higher temperatures (roughly 30000-35000 K). In contrast, the g-mode pulsators have even lower amplitudes (few mmag) and longer periods (30 to $90 \mathrm{~min}$ ) at lower temperatures (roughly 25000-30000 K). In a $\log g-T_{\text {eff }}$ diagram, the hybrids are located at the interface of the $p$-mode and $g$-mode instability regions (see Figure 1 in Lutz et al. 2008a). The class prototypes are EC 14026-2647 (Kilkenny et al. 1997) and PG $1716+426$ (Green et al. 2003), respectively. The known hybrids are HS $0702+6043$ (Schuh et al. 2006), Balloon 090100001 (Oreiro et al. 2005; Baran et al. 2005) and HS 2201+2610 (Lutz et al. 2008b). Asteroseismology has been one of the important tools to constrain the evolutionary history of subdwarf B stars. While an asteroseismological solution provides an instantaneous "snapshot" of the interior stellar structure, extended monitoring of changes over several years in the short, stable $p$-mode pulsation frequencies allows to directly measure evolutionary timescales $\dot{P}$ which can be compared to predictions from evolutionary models. $\dot{P}$ can be measured from O-C diagrams, which will at the same time reveal the presence of potential planets that may in turn have influenced the previous evolution.

\section{EXOTIME}

EXOTIME is the abbreviation of EXOplanet search with the TIming MEthod. This program is led by Roberto Silvotti and Sonja Schuh. Being a collaborative long-term campaign, it involves a substantial number of observers and telescopes all over the world which perform ground-based time-series photometry. Basic information (target objects, observing schedule etc. ) can be found on the program's webpage http://www.na.astro.it/ silvotti/exotime/. While most of the more than 300 exoplanets are found around main sequence host stars, EXOTIME searches planets orbiting evolved sdB pulsators, i.e. extreme horizontal branch objects. Currently, the target list consists of five pulsating sdB stars, HS $0702+6043$ and HS $2201+2610$ being two of them. The search for exoplanets is performed with the timing method or $\mathrm{O}-\mathrm{C}$ analysis, which also allows to derive evolutionary timescales (described on the example of HS $2201+2610$ in a following section). Closely related to the search for exoplanets orbiting sdB stars are evolutionary aspects of sdB stars, late-stage or post RG evolution of planetary systems and the question if planets could be responsible for the extreme mass loss of sdB progenitors.

\section{HS $0702+6043$}

The sdB pulsator HS $0702+6043$ was first identified as a variable in a search program by Dreizler et al. (2002). Its spectroscopic parameters place it at the common boundary of the 


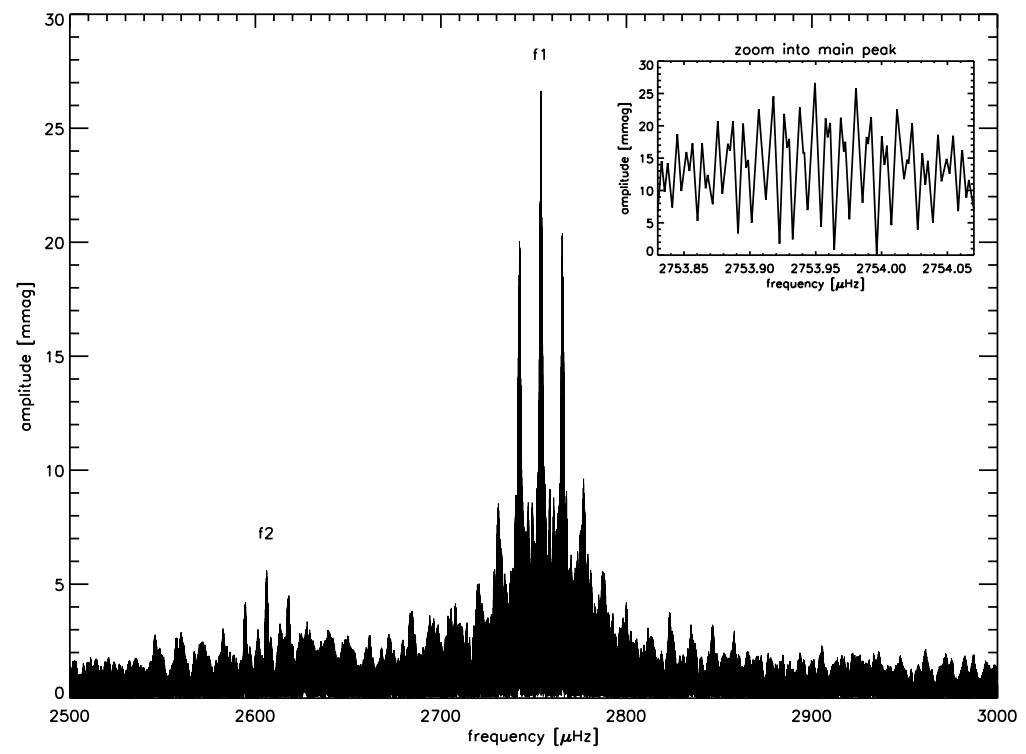

Figure 1: Periodogram of HS $0702+6043$ showing the two main pulsation periods $\mathrm{f} 1$ and $\mathrm{f} 2$. The inset window is a zoom into the main peak.

$p$ - and $g$-mode instability regions and as mentioned above, Schuh et al. (2006) indeed first revealed this object to be a hybrid pulsator. This detection triggered extensive follow-up observations which are listed in Table 1.

There are photometric data available going back to 1999, but unfortunately with large gaps in between. A regular monitoring was not performed until the end of 2007 .

p-modes

The short-period $p$-modes are the relevant ones for the construction of a multi-seasonal O-C diagram. From all the data until Feb 2008 (MB not included), we derived the highresolution frequency spectrum displayed in Figure 1 , which shows the two dominant frequency features f1 and f2 at frequencies of $2753.9 \mu \mathrm{Hz}(363.1 \mathrm{~s})$ and $2606.1 \mu \mathrm{Hz}(383.7 \mathrm{~s})$, respectively. The corresponding amplitudes are 26.6 and $5.5 \mathrm{mmag}$. Due to the large gaps in our data archive, we cannot present a meaningful O-C analysis for HS $0702+6043$ yet, but with a regular EXOTIME monitoring we are confident that this will be possible within the next two years.

\section{g-modes}

The Jan 2005 run (see Table 1) was initiated in order to resolve the low frequency g-mode regime in $\mathrm{HS} 0702+6043$ and indeed, three features could be identified in the data at frequencies of $271.7 \mu \mathrm{Hz}, 318.1 \mu \mathrm{Hz}$ and $206.3 \mu \mathrm{Hz}(61.3 \mathrm{~min}, 52.4 \mathrm{~min}, 80.8 \mathrm{~min})$ with amplitudes of $1.8 \mathrm{mmag}, 1.3 \mathrm{mmag}$ and $0.9 \mathrm{mmag}$, respectively. A periodogram can be found in Lutz et al. (2008a). 


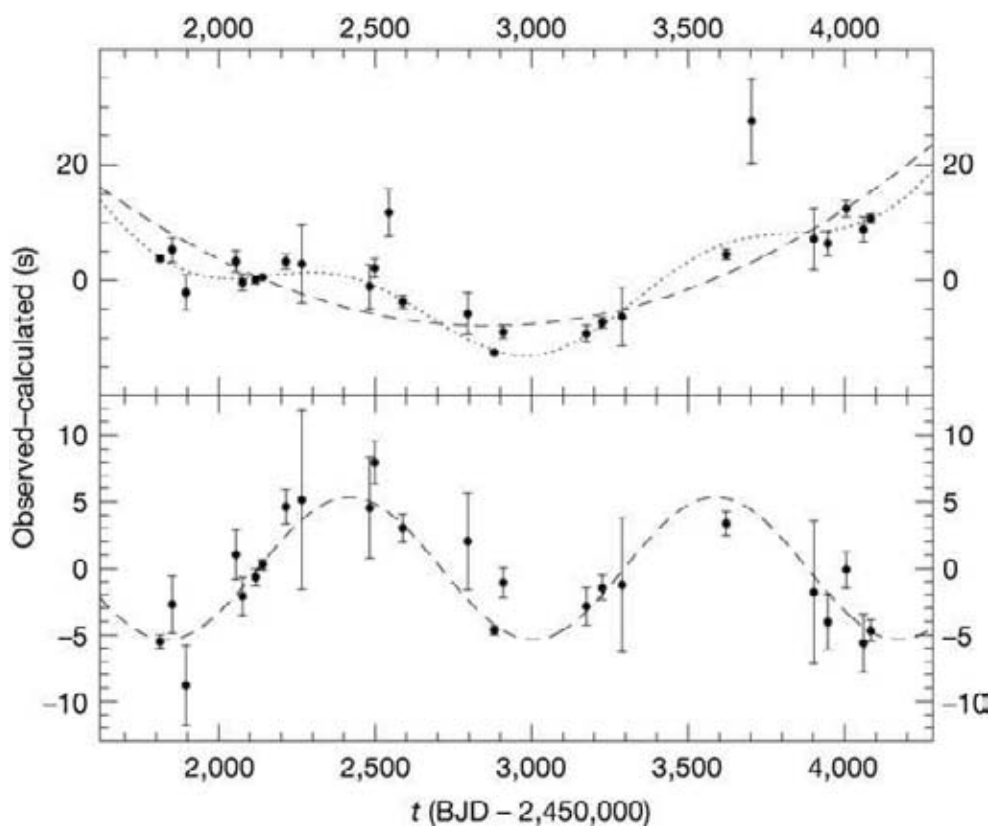

Figure 2: O-C diagram of the main pulsation frequency of HS 2201+2610. Each dot represents a whole campaign. Top panel: parabolic fit, bottom panel: sinusoidal residuals. The sinusoidal component has a semi-amplitude of $5.3 \mathrm{~s}$.

HS $2201+2610$

HS 2201+2610 is so far the only EXOTIME target for which an extended O-C analysis could be executed (Silvotti et al. 2007). Regular data sets are available since 2000.

\section{O-C Analysis}

An O-C (Observed minus Calculated) analysis is a way to measure the phase variations of a periodic function. The observed times of the pulsation maxima (or minima) of the single runs of an observational season are compared to the calculated ephemeris of the whole data set (see e.g. Kepler et al. 1991). Different shapes in the O-C can be identified with different situations: a parabolic shape indicates a linearly changing period, whereas a sinusoidal component can be interpreted by cyclically advanced and delayed timings of the pulsation maxima (or minima) due to the presence of a low-mass companion, which causes the pulsator to wobble around the common barycenter. Figure 2 shows the $\mathrm{O}-\mathrm{C}$ analysis for the main pulsation period of HS 2201+2610, which is around $350 \mathrm{~s}$ at an amplitude of about $10 \mathrm{mmag}$. The parabolic shape of the $\mathrm{O}-\mathrm{C}$ diagram in the top panel of Figure 2 indicates a linearly changing period of $\dot{P}=1.46 \cdot 10^{-12}$. Since this is a positive value, one can infer that this object is expanding, i.e. cooling. The evolutionary timescale can be calculated as $P / \dot{P}=7.6 \mathrm{Myr}$, consistent with theoretical predictions (Charpinet et al. 2002). The bottom panel of Figure 2 displays the sinusoidal residuals which are induced by the gravitational influence of a planetary mass body. Some system parameters that can be derived are the companion's mass of 3.2 Jupiter masses 
(still with an uncertainty due to the unknown inclination), an orbital period of 1170 days, an orbital separation of $1.7 \mathrm{AU}$, a star projected orbital velocity of $99 \mathrm{~m} / \mathrm{s}$ or a planet orbital velocity of $16 \mathrm{~km} / \mathrm{s}$. For more system parameter and a detailed description of the applied assumptions refer to Silvotti et al. (2007).

Acknowledgments. RL thanks the organizers for financial support. The authors thank all observers who contributed observations to the HS 0702+6043 and HS 2201+2610 data archive.

\section{References}

Baran, A., Pigulski, A., Koziet, D., et al. 2005, MNRAS, 360, 737

Charpinet, S., Fontaine, G., Brassard, P., et al. 1997, ApJ, 483, L123

Charpinet, S., Fontaine, G., Brassard, P., \& Dorman, B. 2002, ApJ, 140, 469

Dreizler, S., Schuh, S., Deetjen, J.L., et al. 2002, A\&A, 386, 249

Fontaine, G., Brassard, P., Charpinet, S., et al. 2003, ApJ, 597, 518

Green, E.M., Fontaine, G., Reed, M.D., et al. 2003, ApJ, 583, L31

Jeffery, C.S., \& Saio, H. 2006, MNRAS, 372, L48

Kepler, S.O., Winget, D.E., Nather, R.E., et al. 1991, ApJ, 378, 45

Kilkenny, D., Koen, C., O'Donoghue, D., \& Stobie, R.S. 1997, MNRAS, 285, 640

Lutz, R., Schuh, S., Silvotti, R., et al. 2008a, ASPC, 392, 339

Lutz, R., Schuh, S., Silvotti, R., et al. 2008b, A\&A, submitted

Oreiro, R., Pérez Hernández, F., Ulla, A., et al. 2005, A\&A, 438, 257

Schuh, S., Huber, J., Dreizler, S., et al. 2006, A\&A, 445, L31

Silvotti, R., Schuh, S., Janulis, R., et al. 2007, Nature, 449, 189

\section{DISCUSSION}

Kepler: Can you show the (O-C) values for the data?

Lutz: So far, we only have a crude, preliminary (O-C) values without any applied weights. We hope that weighting the different runs with different qualities will improve the results. Hopefully, we can give you more details in a few months. 


\title{
Mode identification in rapidly pulsating subdwarf B stars from monochromatic amplitude- and phase variations
}

\author{
S. K. Randall ${ }^{1}$, V. van Grootel ${ }^{2}$, G. Fontaine ${ }^{3}$, S. Charpinet $^{2}$, and P. Brassard ${ }^{3}$ \\ ${ }^{1}$ ESO, Karl-Schwarzschild-Str. 2, 85748 Garching bei München, Germany \\ 2 Laboratoire d'Astrophysique de Toulouse-Tarbes, Université de Toulouse, \\ CNRS, 14 av. E. Belin, 31400 Toulouse, France \\ ${ }^{3}$ Département de Physique, Université de Montréal, CP 6128, \\ Succursale Centre-Ville, Montréal, QC H3C 3J7, Canada
}

\begin{abstract}
We present a mid-term report on a project aimed at partially identifying the main modes of pulsation in the rapidly pulsating subdwarf B star PG $1047+003$. Using the unique HIT-MS mode on FORS2 at the VLT we obtained $\sim 3000$ spectra spread over 4 consecutive half-nights with a typical sampling rate of $20 \mathrm{~s}$. These will be used to extract monochromatic pulsation amplitudes and phases that can be fit to model predictions in order to determine the degree $\ell$ of the mode in question.
\end{abstract}

Individual Objects: PG $1047+003$

\section{Introduction}

Subdwarf B (sdB) stars are hot $\left(20,000 \leq T_{\text {eff }} \leq 40,000 \mathrm{~K}\right)$, compact $(5.0 \leq \log g \leq 6.2)$, evolved helium-core burning objects located on the extreme horizontal branch (EHB) of the $\mathrm{H}-\mathrm{R}$ diagram. It is thought that sdB progenitors lost almost all their hydrogen envelopes near the tip of the red giant branch and thus settled on the EHB rather than on the usual horizontal branch or in the red clump.

While a number of evolutionary scenarios have been proposed and simulated numerically (e.g. Han et al. 2002, 2003), they remain to be tested observationally. A good way of doing this is by determining the mass and hydrogen-thickness distribution of known sdB pulsators from asteroseismology, since these depend sensitively on the formation channels. Asteroseismic analyses leading to the determination of the targets' fundamental parameters (including the mass and hydrogen shell thickness) have so far been carried out for 12 fast pulsators among sdB's, and first evolutionary trends are emerging (see Fontaine et al. 2008 for a recent review).

\section{Methodology}

In contrast to many other types of pulsators known, a priori mode identification does not always seem to be necessary for the successful asteroseismology of the rapidly pulsating socalled EC 14026 stars (van Grootel et al. 2008). However, independent constraints on mode identification nevertheless provide a nice consistency check for the validity of the asteroseismic solutions found, and for some pulsating sdB stars, it may be necessary to discriminate 


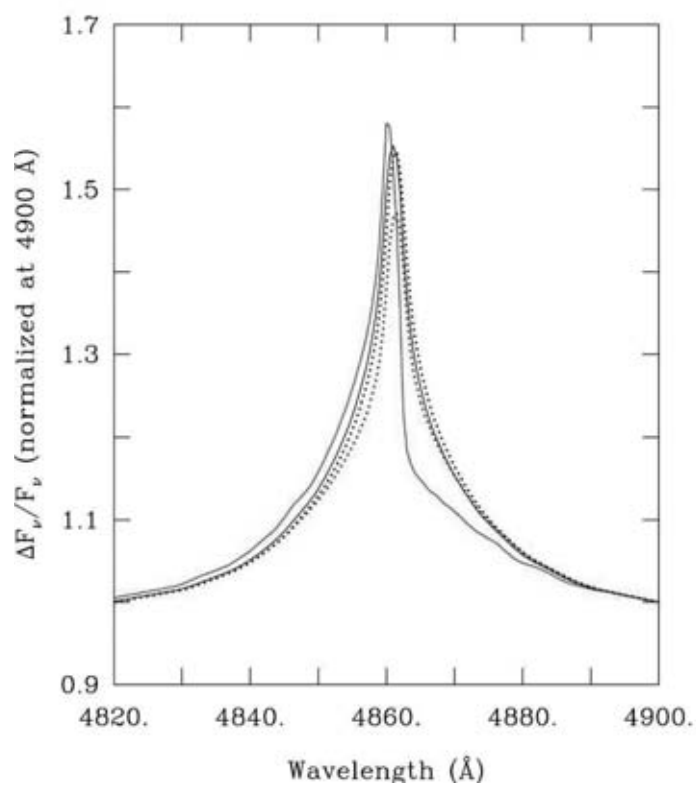

Figure 1: Predicted pulsational amplitude variation across the $\mathrm{H} \beta$ line for a model with the atmospheric parameters of PG $1047+003$. The black curves refer to a degree index $\ell=1$, while the red (grey) ones indicate $\ell=2$. For both cases, the dotted lines were computed assuming no radial velocity variations, whereas for the continuous curves a radial velocity variation of $10 \mathrm{~km} / \mathrm{s}$ was assumed.

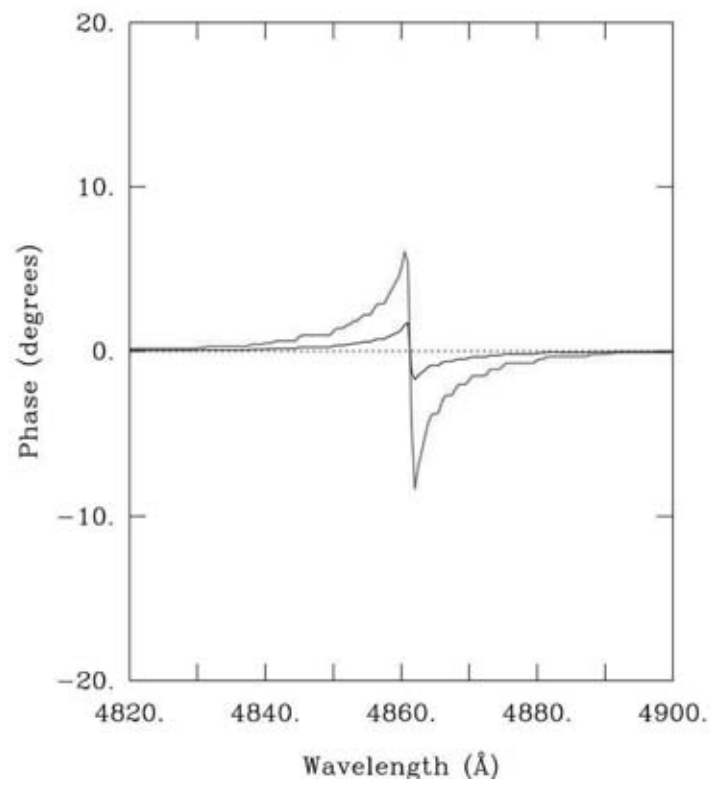

Figure 2: As Figure 1, only for phase variations across the line. 
between models that match the observed periodicities equally well. Partial mode identification for subdwarf $B$ stars has so far been performed through the exploitation of multi-colour photometry (e.g. Tremblay et al. 2006), the line-profile variations observed during a pulsation cycle (e.g. Telting et al. 2008), and rotational splitting induced for the case of a rapid rotator (e.g. Charpinet et al. 2005), with varying success. While the first two methods can, in principle, be applied to all pulsating subdwarf B stars, their mode identification power is in practice severely limited by the $\mathrm{S} / \mathrm{N}$ of the observations.

The aim of this project is to test the mode identification potential of time-resolved lowresolution spectroscopy. Essentially an extended version of the multi-colour method, the technique proposed makes use of the strong signature of the degree index $\ell$ on the amplitudes and phases in the spectral lines, which is lost when analysing wavelength-integrated data. It was quite successfully applied to the ZZ Ceti white dwarf G29-38 (Clemens et al. 2000). We recently incorporated radial velocity variations into our white dwarf and sdB model atmosphere codes, which were previously used only for the mode identification of oscillations observed with multi-colour photometry (see Randall et al. 2005). This upgrade allows us to accurately model the monochromatic amplitude and phase variations expected across the spectral lines for different pulsation modes. Figures 1 and 2 respectively show the resulting amplitude and phase variations across the $\mathrm{H} \beta$ line for a subdwarf $\mathrm{B}$ star model with the atmospheric parameters of PG $1047+003$ (see below) for modes with $\ell=1$ (black) and $\ell=2$ (red). Looking at the plots, it is particularly the changes in phase across the line profile that appear to hold significant discriminative power with respect to the degree index of the mode in question.

\section{PG $1047+003$}

PG $1047+003$ is a relatively bright $(V=13.47)$, apparently single, rapidly pulsating subdwarf $B$ star with 16 confirmed independent periods in the 90-162 s range at relatively low amplitudes below 10 mmags (Billères et al. 1997; O'Donoghue et al. 1998; Kilkenny et al. 2002). In accordance with the published values, our own analyses of high $\mathrm{S} / \mathrm{N}$ spectra obtained on the MMT and the Kitt Peak 90" telescope (E.M. Green, private communication) indicate atmospheric parameters $T_{\text {eff }} \sim 35,000 \mathrm{~K}$ and $\log g \sim 5.82$, placing PG 1047+003 towards the high temperature and gravity end of the EC 14026 instability strip. Incidentally, PG 1047+003 was one of the first sdB stars to be submitted to a preliminary asteroseismological analysis (Charpinet et al. 2003). We attempted a more extensive search in parameter space using an updated code (see e.g. van Grootel et al. 2008 for more details on this), and found 4 solutions that are in agreement with spectroscopic estimates of the effective temperature and surface gravity. However, none of these are entirely convincing from a mode identification point of view: because of the observed mode density, it is very difficult to find a model with a good period match that does not include at least 2 modes with $\ell=3$. The latter are thought to have extremely low amplitudes when integrated over the visible disk of the star, and should not be readily detectable (Randall et al. 2005). Therefore, it makes sense to place independent constraints on the degree indices of the observed periodicities in order to solve the mystery. This is precisely what we will attempt with the data presented in the next section.

\section{Observations}

We obtained low-resolution time-series spectroscopy of PG 1047+003 using the unique HITMS mode of FORS2 mounted at VLT-UT1 in Chile. Rarely used and not very well publicized, the HIT mode allows the acquisition of fast time-series photometry or spectroscopy with virtually no dead time in between frames. It overcomes the "problem" of the readout time by exposing only a small part of the chip and successively shifting the exposed blocks of rows across the CCD while already integrating on the next spectrum. This way, 42 events 


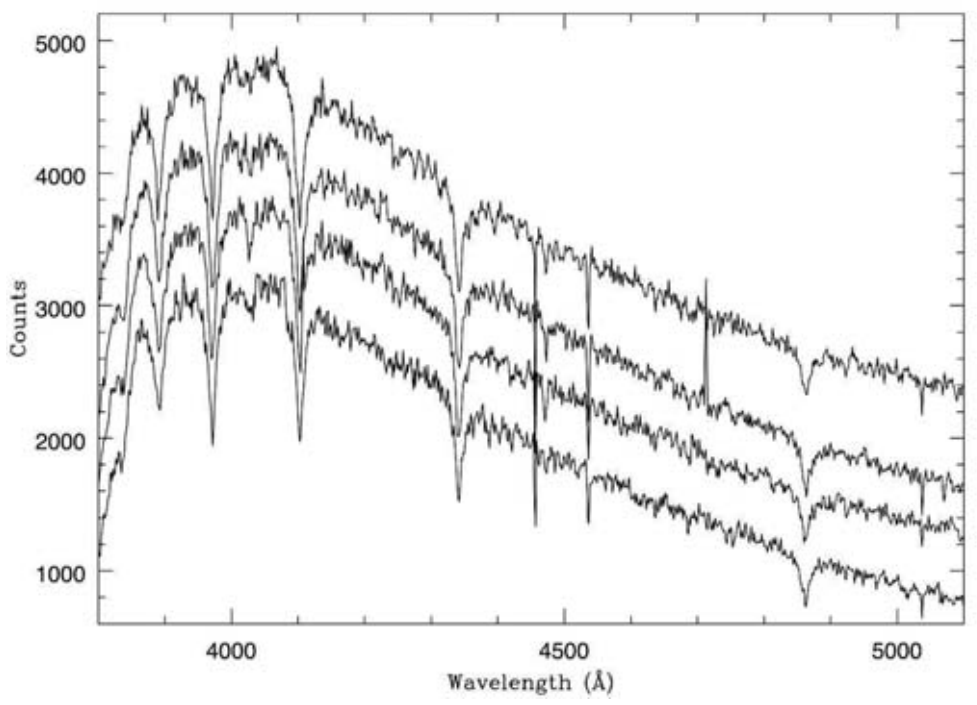

Figure 3: Typical individual extracted and wavelength calibrated spectra of PG $1047+003$ as obtained with FORS2 in HIT-MS mode setting the exposure time to $20 \mathrm{~s}$. The upper spectra have been arbitrarily shifted in the $y$-direction for visualisation purposes; the $\mathrm{S} / \mathrm{N}$ of the individual spectra shown is around 40 . Note that some residual instrumental effects appear to remain since the data reduction products shown are only preliminary.

can be recorded per 40-s readout of the chip. These events may be either simple imaging, low-resolution spectra, or even pairs of target and comparison star spectra. In spectroscopy mode, the wavelength ranges from 3300-6012 $\AA$ (grism 600B) or 6000-11,000 $\AA$ (grism $3001)$ can be covered at low resolution.

We were allocated 4 half-nights of observing time. Of these, we lost a few hours to clouds, but apart from that conditions were mostly clear with the seeing varying between 0.6 " and 1.6". In total, we accumulated 2976 spectra with an integration time of $20 \mathrm{~s}$ and a wavelength resolution of $\sim 3.5 \AA$ at $4500 \AA$. Figure 3 shows 4 consecutive spectra obtained on our third night of observation; the sequence shown therefore has a total time baseline of $80 \mathrm{~s}$, similar to the shortest pulsations detected for PG 1047+003. From a visual inspection of the plot there appear to be no systematic variations from one spectrum to the next that could be attributed to the oscillations. However, these are likely too weak to be seen and will be uncovered only from careful Fourier analysis, which is the next step planned.

\section{Conclusions}

Using the unique HIT-MS mode offered on FORS2 at the VLT, we obtained a large number of fast time-series spectra of the rapidly pulsating subdwarf B star PG $1047+003$. While there is no immediate evidence of pulsation in the reduced spectra, the main modes of oscillation will likely be uncovered using more sophisticated techniques since the $\mathrm{S} / \mathrm{N}$ of the individual measurements is relatively high. We are hopeful that this will eventually yield constraints on the degree indices of the dominant modes of pulsation. 
Acknowledgments. We would like to acknowledge the help of the science operations staff at Paranal, in particular that of Kieran O'Brien, who gave us advice on the best observing strategy to pursue with the rather complicated HIT-MS mode.

\section{References}

Billères, M., Fontaine, G., Brassard, P., et al. 1997, ApJ, 487, L81

Charpinet, S., Fontaine, G., Brassard, P., et al. 2005, A\&A, 443, 251

Charpinet, S., Fontaine, G., \& Brassard, P. 2003, NATO Sciences Series II, 105, 69

Clemens, J. C., van Kerkwijk, M. H., \& Wu, Y. 2000, MNRAS, 314, 220

Fontaine, G., Brassard, P., Charpinet, S., et al. 2008, ASPC, 392, 231

Han, Z., Podsiadlowski, P., Maxted, P. F. L., et al. 2002, MNRAS, 336, 449

Han, Z., Podsialowski, P., Maxted, P. F. L., \& Marsh, T. R. 2003, MNRAS, 341, 669

Kilkenny, D., Billères, M., Stobie, R. S., et al. 2002, MNRAS, 331, 399

O'Donoghue, D., Koen, C., Lynas-Gray, A. E., et al. 1998, MNRAS, 296, 306

Randall, S. K., Fontaine, G., Brassard, P., \& Bergeron, P. 2005, ApJS, 161, 456

Telting, J. H., Østensen, R. H., Geier, S., et al. 2008, A\&A, ASPC, 392, 301

Tremblay, P.-E., Fontaine, G., Brassard, P., et al. 2006, ApJS, 165, 551

van Grootel, V., Charpinet, S., Fontaine, G., et al. 2008, A\&A, 488, 685

\section{DISCUSSION}

Kepler: Did you do your flux calibration?

Randall: Not yet, however we did obtain spectra for a specphot standard and plan to flux calibrate the data in the future.

Dupret: For feasible mode identification you need atmosphere models appropriate for your type of stars. Could you precise which atmosphere models you use and their validity in this context? The same question applies also for the limb darkening law.

Randall: We use our own local sdB model atmospheres incorporating hydrogen and helium (but no metals) and non-LTE effects. On the basis of these, we compute our own specific intensities and their derivatives with respect to $\log g$ and $\log T_{\text {eff }}$

Noels: You said that mode identification was not unnecessarily a main goal of asteroseismology. It is probably true for sdBs but not the case for other variable stars like SPB and $\beta$ Cep stars, especially since we never have an infinite accuracy in the effective temperature

Randall: I completely agree. I simply wanted to point out that, in a number of cases, sdB asteroseismology does not rely on a priori mode identification. However, mode identification using an independent means can provide an important consistency check for the asteroseismic solution found, and is certainly an interesting exercise, even for rapidly pulsating subdwarf B stars. 